\title{
Fluctuations of the Magnetization in Thin Films due to Conduction Electrons
}

\author{
A. Rebei ${ }^{1 *}$ and M. Simionato ${ }^{2}$ \\ 1. Seagate Research Center, Pittsburgh, Pennsylvania 15222,USA \\ 2. Department of Physics and Astronomy, \\ University of Pittsburgh, Pennsylvania 15260, USA
}

(Dated: 12-10-2004)

\begin{abstract}
A detailed analysis of damping and noise due to a $s d$-interaction in a thin ferromagnetic film sandwiched between two large normal metal layers is carried out. The magnetization is shown to obey in general a non-local equation of motion which differs from the the Gilbert equation and is extended to the non-adiabatic regime. To lowest order in the exchange interaction and in the limit where the Gilbert equation applies, we show that the damping term is enhanced due to interfacial effects but it also shows oscillations as a function of the film thickness. The noise calculation is however carried out to all orders in the exchange coupling constant. The ellipticity of the precession of the magnetization is taken into account. The damping is shown to have a Gilbert form only in the adiabatic limit while the relaxation time becomes strongly dependent on the geometry of the thin film. It is also shown that the induced noise characteristic of sd-exchange is inherently colored in character and depends on the symmetry of the Hamiltonian of the magnetization in the film. We show that the sd-noise can be represented in terms of an external stochastic field which is white only in the adiabatic regime. The temperature is also renormalized by the spin accumulation in the system. For large intra-atomic exchange interactions, the Gilbert-Brown equation is no longer valid.
\end{abstract}

PACS numbers: 75.10.Jm, 75.30.Gw, 76.60.Es 


\section{INTRODUCTION}

The need for ever higher storage densities and faster retrieval data rates in magnetic recording is bringing out new fundamental physical challenges to the industry. In any physical device, the main issue is the signal to noise ratio (SNR). For nano-devices, we expect a decrease in the signal output and an increase in the noise. Therefore any simple scaling-down of the current devices is bound to fail. Hence the need for an understanding of the noise source in these devices so that novel solutions to the SNR problem can be devised. In magnetic transition-metal based devices, the conduction electrons are one such source of noise. The stochastic Landau-Lifshitz-Gilbert equation, which for short we will call the Gilbert-Brown equation $(\mathrm{GB})^{1,2}$, has been the main tool in studying the noise at the phenomenological level. ${ }^{3}$ The GB equation has been quite successful in predicting the right form for the damping term for most of the measurements in this area. The noise term, which is represented by a stochastic term, is however very qualitative since any meaningful account of the noise in a magnetic system is dependent on the microscopic mechanisms that give rise to the damping term in the first place. The GB equation has the simple form

$$
\frac{d \mathbf{S}}{d t}=\mathbf{S} \times\left(\mathbf{H}_{e f f}+\alpha \frac{\mathbf{d} \mathbf{S}}{d t}+\mathbf{h}\right)
$$

with $\mathbf{H}_{\text {eff }}$ the effective field, $\alpha$ is a damping constant and the stochastic field $\mathbf{h}(t)$ satisfies

$$
\left\langle h_{i}(t) h_{j}\left(t^{\prime}\right)\right\rangle=2 \alpha k_{B} T \delta_{i j} \delta\left(t-t^{\prime}\right) .
$$

Given that more details about the physics of the devices are now needed to better control them, a more microscopic treatment of the noise is in order. This is the subject of this paper. However, we will not be able to treat this question in its full generality simply because specific details about all systems of interest differ from one to the other. In this study, we focus mainly on a thin magnetic film geometry embedded between two normal conductors. Such a geometry happens in , e.g., a read-head in a recording device and it is also widely used in spin-momentum transfer problems. Damping and noise due to conduction electrons is expected to be of importance in these systems. To study this case, we need to compute the effective action for magnons in real time for a thin magnetic film embedded in a conducting paramagnet. We will derive and solve the corresponding equations of motion, then we will discuss the noise spectrum. This program has already been carried out successfully in an 
earlier paper ${ }^{4}$, where an exactly solvable Caldeira-Leggett-like model has been discussed. In that simple case we were able to compute the effective action exactly. However, the model of ref. 4 is quite phenomenological and not very predictive, since it involves a very large freedom in the choice of the coupling constants. The noise expressions in 4 will however surface again in our discussion in the adiabatic limit.

In more realistic microscopic models, the effective action cannot be computed exactly, nevertheless it can be computed in an approximate way. In this paper we will discuss a very simple microscopic model, which still contains the basic physics of electrons and magnons in thin films and presents quite non-trivial features. This model has been investigated previously by many authors, however the relevant references discussing the physics of thin films that are directly related to the work presented here are 5-11. These latter works are primarily interested in effects similar to the spin-momentum transfer problem of Slonczewski ${ }^{12}$ who studied the influence of a nonzero polarized current on the dynamics of the magnetization in thin film multilayers. In this paper, we mainly focus on the single film case with and without a biased voltage. The case with a polarized current and non-collinear magnetization will be briefly treated numerically in the last section. We will be mainly studying the finite size effects of the film on the dynamic of the magnetization but our method will allow us also to address questions related to the validity of the GB in the atomistic regime, an area of growing interest in recording physics. ${ }^{13,14}$

In the presence of a s-d interaction, the conduction electrons exercise an effective torque on the local magnetic moment which can be put in a stochastic form. Therefore, in addition to the usual thermal magnetic noise, there will be an additional component due to the conduction electrons and one of our tasks is to find out when this contribution can be absorbed in the usual Gilbert damping term . The origin of damping in ferromagnets is still an open problem. In iron, it is believed that the conduction electrons through the exchange interaction are the main channel for the dissipation. ${ }^{15}$ In Nickel and Cobalt, the spin-orbit coupling is suggested to be the mechanism for the dissipation. ${ }^{16,17}$ The calculations of 15 and 16 are however not totally self-consistent; an adjustable parameter, the relaxation of conduction electrons to the lattice, is needed for a meaningful result for the dissipation and no treatment of noise has been attempted. Both mechanisms give a Gilbert-form for the damping. In this paper we study damping in thin films within the sd-model of ref. 15. It has been recently argued that the damping should reflect the geometry of the sample 
and hence the damping should have a non-Gilbert tensor form. ${ }^{18}$ The linear model treated in ref. 4 showed that the symmetry of the Hamiltonian has no effect on the damping. In this paper, we treat a non-linear interaction between the conduction electrons and the magnetization, the sd-exchange, and show that in this case the damping is sensitive to the symmetry of the Hamiltonian only for high frequencies. Therefore for the macroscopic average magnetization of the sample, the Gilbert damping is correct. Symmetries are important only for microscopic magnetization.

As a result of the recent illuminating work of Simanek ${ }^{11}$, our work will turn out also to be intimately related to the spin-pumping theory of Tserkovnyak, Brataas and Bauer $(\mathrm{TBB})^{6}$ that treated similar questions using scattering theory. Their damping is nicely expressed in terms of the mixing conductance, a quantity that needs to be computed by ab-initio calculations. In this work we use a very different method which will enable us to treat simultaneously atomic magnetic moments and macroscopic magnetic moments simultaneously. Moreover, we will be able to give explicit expressions for the damping and noise at all frequencies and include finite size-effects of the film within the sd-model. Realistic systems can also be treated by this method but will require numerical computations. Hence our results will be of interest to those interested in atomic simulations of magnetic systems, an area which is starting to become important for magnetic recording. ${ }^{14}$

The experimental work of Covington et al. on spin momentum transfer is another motivation for our work. ${ }^{19}$ This latter work showed that in a biased spin valve with currents below the critical current, i.e. current needed to switch the thin layer, the resistance shows large $1 / \mathrm{f}$-type noise in the $\mathrm{MHz}-\mathrm{GHz}$ regime. Our system is similar to a spin valve except that we do not have a reference layer. This will enable us to examine the contribution of the sd-exchange to the line-width in the $\mathrm{N} / \mathrm{F} / \mathrm{N}$ structure and the spin momentum transfer. A full micromagnetic treatment is also given that includes the effect of non-spin flipping events on the spin-momentum transfer. Our conclusions will be helpful to the interpretation of the experiment and the micromagnetic calculations. We will show an example where the noise has its origins in the non-uniformity of the in-plane magnetization.

The paper is organized as follows. In sect. II, we set up our notation and the Hamiltonian used in our calculations. We use a non-isotropic Hamiltonian that takes into account the ellipticity of the magnetization which is typical in thin magnetic films or local magnetic moments. Using the real-time formalism for our model, we first compute the free propa- 
gators of the theory. Then, we derive the effective action of the system by integrating out the electron degrees of freedom. We derive a stochastic equation for the magnetic moment that is different from the GB equation. These equations are non-local in space and in time. These equations will be especially needed in atomic-type simulations of magnetic systems where the local effective field is large compared to macroscopic fields. In sect. III, we discuss the limits under which we can recover the GB equation for this model in a macroscopic system $\mathrm{N} / \mathrm{F} / \mathrm{N}$. We show that interfaces enhance the damping and the fluctuations of the magnetization. The bulk damping is assumed to be due to conduction electrons interacting with the lattice. In sect. IV, we show how to calculate the noise spectrum of the magnetization and discuss its dependencies on the anisotropy, initial conditions and on the spin accumulation for the $\mathrm{N} / \mathrm{F} / \mathrm{N}$ case. Moreover, we show that in the adiabatic limit this sd-exchange interaction is equivalent to a stochastic external field with a Gaussian white noise distribution and effective temperature that reflects the geometry of the system. For high frequencies, we show that the GB equation is no longer valid and that the damping reflects the symmetry of the Hamiltonian which does not appear in the linear or adiabatic regime. In sec. V, we discuss a geometry similar to that of ref. 19 using a macroscopic spin transfer model. Based on the quantum calculations in previous sections and the micromagnetic calculations, we suggest that the noise in 19 is due to thermally assisted transitions between two non-uniform states of the magnetization. Finally in the appendix, we give various expressions needed in the calculation of the correlation functions of the magnetic moment and discuss the dependence of the damping on the symmetry of the Hamiltonian of the magnetic moment.

\section{HAMILTONIAN FORMULATION AND THE ONE-LOOP EFFECTIVE AC- TION}

Let us consider a thin film of magnetic material interacting with a large external magnetic field of order one Tesla or more $\mathbf{H}=(0,0, H)$, constant in time, uniform in space and directed along the $z$ direction which is in-plane. Let us assume the film has linear dimensions $D \times L_{y} \times L_{z}$, i.e. it has a rectangular section in the $y z$ plane (the plane parallel to the magnetic field), with area $L_{y} L_{z}$ and thickness $D$ in the $x$ direction, with $D<<L_{y}$ and $D<<L_{z}$, as 
shown in figure 1. The magnetic film will be later assumed to be sandwiched between two

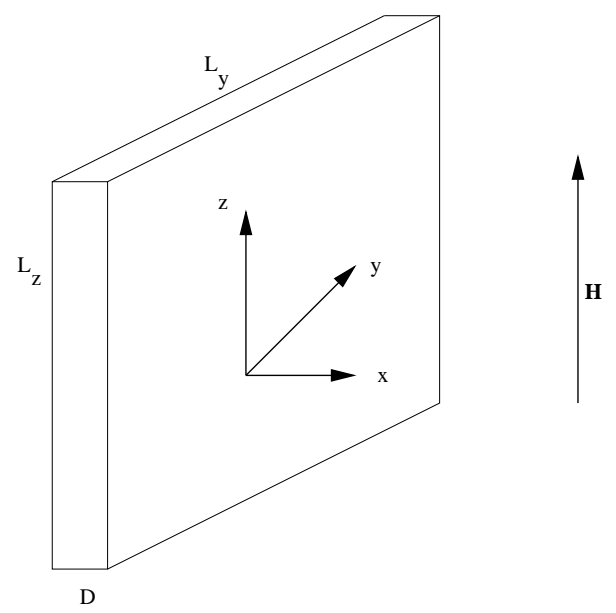

FIG. 1: The geometry of the thin film described in the text.

large normal conductors. We are interested in studying the effects of the exchange magnetic field due to conduction electrons on the average magnetization of the thin film as well as the local atomic moments. Ultimately, we will be interested in studying the case $D$ is much smaller than the lateral dimensions of the film, i.e. the thin film limit. The finite size effects related to the breaking of translation invariance in the $x$ direction will be of primary interest to us since in this case the effect of the conduction electrons on the average magnetization is strongest. A path integral formulation proves to be very useful in problems of this sort.

A ferromagnetic material is roughly a system of quasi-free electrons of spin $\mathbf{s}$ (the $4 \mathrm{~s}$ electrons) interacting with bound electrons of spin $\mathbf{S}$ (the 3 d electrons) via an Heisenberg Hamiltonian

$$
\mathcal{H}_{\text {spin-int }}=-\frac{J}{2} \int_{V} d^{3} x \psi^{\dagger}(\mathbf{x}) \vec{\sigma} \psi(\mathbf{x}) \cdot \mathbf{S}(\mathbf{x}),
$$

where $J$ is the interaction constant, of the order of at least $0.1 \mathrm{eV}$ in the macroscopic case and about $10.0 \mathrm{eV}$ in the microscopic case, $\vec{\sigma}=\left(\sigma_{1}, \sigma_{2}, \sigma_{3}\right)$ is the vector with components the Pauli matrices and $\psi$ is the 2-component electron field. The $3 \mathrm{~d}$ bound electrons are mostly aligned with the external magnetic field and they are the source for the magnetization $\mathbf{S}$ of the material. The Hamiltonian of the conduction electrons is then given by

$$
\mathcal{H}_{e}=\int_{V} d^{3} x \psi_{\alpha}^{\dagger}(\mathbf{x})\left[-\frac{\nabla^{2}}{2 m}+V(\mathbf{x})\right] \psi_{\alpha}(\mathbf{x})+\mathcal{H}_{\text {spin-int }}
$$




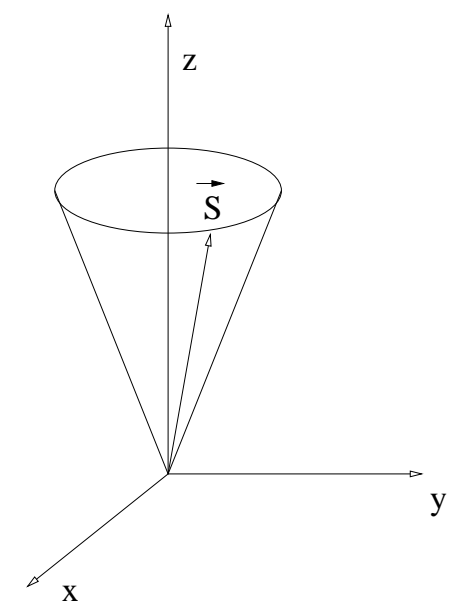

FIG. 2: The local magnetization vector.

where the potential $V(\mathbf{x})$ is a spin independent confining potential of the structure. For a large external field $H$, the $S^{z}$-component of the magnetization can be taken to be a constant and hence its interaction can be absorbed in the diagonal part of the energy.

The simplest possible effective Hamiltonian we can construct for the magnetization is

$$
\mathcal{H}_{\text {spin }}=\sum_{c} \mathcal{H}_{\text {spin }, c}=\int_{V} d^{3} x\left[-\mathbf{H} \cdot \mathbf{S}+\frac{1}{2} c_{x x} S^{x}(\mathbf{x})^{2}+\frac{1}{2} c_{y y} S^{y}(\mathbf{x})^{2}\right] .
$$

where $c$ is the cell index with volume $v=V / N$. In micromagnetic simulations, $v$ is of the order of $10 \mathrm{~nm}^{3}$ while in atomistic calculations it is of the order of the $0.4 \mathrm{~nm}^{3}$. We have neglected higher powers of the $S_{c}^{x}$ and $S_{c}^{y}$ components. This can be justified once we notice that in a magnetic material the spins are mostly oriented in the direction of the magnetic field, i.e. the spin vector has locally $x$ and $y$ components which are small with respect to the $z$ component:

$$
\mathbf{S}_{c}=\left(S_{c}^{x}, S_{c}^{y}, S_{c}^{z}\right), \quad\left|S_{c}^{x}\right|,\left|S_{c}^{y}\right| \ll S_{c}^{z}
$$

In other words, in this paper we will consider the case in which the spin vector has a small angle with the $z$ axis (see Figure 2). Micromagnetic calculations show that an angle of 20 degrees can still be considered small . The dynamics of large angles and in particular the possibility of magnetization switching is quite interesting too, but it cannot be addressed within the approximations used here. ${ }^{20}$

The advantage of the small angle approximation, i.e. taking $S_{c}^{z}$ time-independent, is the 
simplification of the commutation relations, $\mathbf{S} \times \mathbf{S}=i \mathbf{S},{ }^{21,22}$ which become

$$
\left[S_{c}^{x}, S_{c^{\prime}}^{y}\right]=i S_{c}^{z} \delta_{c c^{\prime}}
$$

This implies that $S_{c}^{y}$ is canonically conjugate to $S_{c}^{x}$. We neglect any exchange stiffness between the cells. Next, we consider the case of an infinite wavelength spin wave with wave-vector $k=0$. This corresponds to an homogeneous spin field. The Heisenberg equation of motion can be derived from the Hamiltonian and the commutation relation and they assume the form

$$
\dot{S}_{x}=c_{y y} S_{y}, \quad \dot{S}_{y}=-c_{x x} S_{x}
$$

The coefficients $c_{x x}$ and $c_{y y}$ are related to the anisotropies of the medium and we are especially interested in the strongly anisotropic case, $c_{x x} \gg c_{y y}$. The Heisenberg equations of motion can be trivially solved in the form

$$
\left(\begin{array}{l}
S_{x}(t) \\
S_{y}(t)
\end{array}\right)=e^{\mathcal{M}\left(t-t_{0}\right)}\left(\begin{array}{c}
S_{x}(t) \\
S_{y}(t)
\end{array}\right)
$$

where $\mathcal{M}$ is the matrix

$$
\mathcal{M}=\left(\begin{array}{cc}
0 & c_{y y} \\
-c_{x x} & 0
\end{array}\right)
$$

More explicitly, we have

$$
\begin{aligned}
& S_{x}(t)=\cos \omega_{0}\left(t-t_{0}\right) S_{x}\left(t_{0}\right)+\sqrt{\frac{c_{y y}}{c_{x x}}} \sin \omega_{0}\left(t-t_{0}\right) S_{y}\left(t_{0}\right), \\
& S_{y}(t)=-\sqrt{\frac{c_{x x}}{c_{y y}}} \sin \omega_{0}\left(t-t_{0}\right) S_{x}\left(t_{0}\right)+\cos \omega_{0}\left(t-t_{0}\right) S_{y}\left(t_{0}\right),
\end{aligned}
$$

where $\omega_{0}=\sqrt{c_{x x} c_{y y}}$ is the frequency of the elliptic precession of the magnetization. In the rest of the paper, we calculate the effect of the conduction electrons on this solution in both low and high frequency limits. Since the interaction with the non-dynamical $S^{z}$ component has been already accounted for in the Hamiltonian of the conduction electrons, only the $\sigma_{1}$ and $\sigma_{2}$ terms enter in the interaction with the dynamical spin field. In momentum space this interaction term reads

$$
\mathcal{H}_{i n t}=-\frac{J}{2 N^{1 / 2}} \sum_{\mathbf{q k}}\left[a_{\mathbf{q}-\mathbf{k}}^{\dagger} \sigma_{1} a_{\mathbf{q}} S_{\mathbf{k}}^{x}+a_{\mathbf{q}-\mathbf{k}}^{\dagger} \sigma_{2} a_{q} S_{\mathbf{k}}^{y}\right]
$$

The interaction Hamiltonian $\mathcal{H}_{\text {int }}$ commutes with the total Hamiltonian. This means that only two interactions are possible: a) a spin up electron $+1 / 2$ emits a +1 magnon 
and becomes a spin down $-1 / 2$ electron; b) a spin down $-1 / 2$ electron absorbs a spin +1 magnon and becomes a spin up $+1 / 2$ electron. These interactions are represented in figure 3.

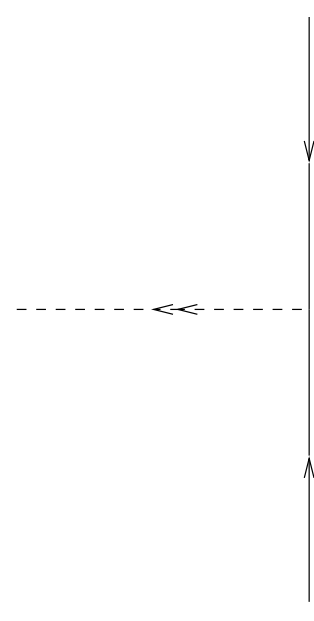

a)

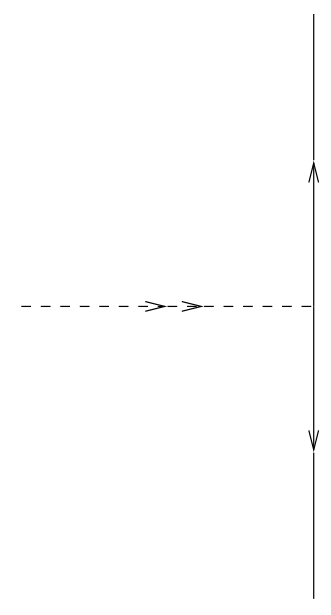

b)

FIG. 3: Conservation of angular momentum in the $z$ direction

Next, we calculate the effective action of the theory to first order in $J^{2}$. This is done by eliminating the electronic degrees of freedom after which, we get a stochastic equation for the magnetization valid in the non-adiabatic regime.

First we compute the free propagators which are obtained by inverting the diagonal differential operators $i \partial_{t}-\mathcal{E}_{k}$ for the electron field and the differential operator

$$
\mathcal{D}_{i j}^{-1}=\left.\frac{\delta \mathcal{S}}{\delta S_{-k}^{i}(t) \delta S_{k}^{j}\left(t^{\prime}\right)}\right|_{\phi=0}=-\left(\begin{array}{cc}
c_{x x} & \partial_{t} \\
-\partial_{t} & c_{y y}
\end{array}\right) \delta_{P}\left(t-t^{\prime}\right)
$$

for the spin field. Here $\mathcal{E}_{k}$ and $\mathcal{S}$ are the diagonal parts of the action for the electrons and the magnetization, respectively. Therefore, one has to solve the differential equations for the corresponding propagators $\mathcal{G}_{P}$ and $\mathcal{D}_{P}$ :

$$
\left(i \partial_{t}-\mathcal{E}_{k}\right) \mathcal{G}_{P}\left(t, t^{\prime}, k\right)=\delta_{P}\left(t-t^{\prime}\right)
$$

and

$$
\mathcal{D}^{-1} \mathcal{D}_{P}\left(t, t^{\prime}, k\right)=\delta_{P}\left(t-t^{\prime}\right)
$$

where the delta function is defined on the closed-time path (CTP) $P$ and the boundary conditions are such that the spin field is periodic on the path $\mathrm{P}$ while the electron field is 
anti-periodic. ${ }^{23}$ Notice that eq. 10 is a $2 \times 2$ matrix in the spin space whereas eq. 11 is a $2 \times 2$ matrix in the complex plane. The first equation is diagonal and can easily be solved with solution

$$
\mathcal{G}_{P}\left(t-t^{\prime}\right)=-i e^{-i \omega_{k}\left(t-t^{\prime}\right)} \Theta_{P}\left(t-t^{\prime}\right)+A e^{-i \omega_{k}\left(t-t^{\prime}\right)}
$$

where the matrix $A$ is the integration constant corresponding to a generic solution of the homogeneous equation. The boundary condition $\mathcal{G}_{P}\left(t_{0}, t^{\prime}\right)=-e^{\beta \mu} \mathcal{G}_{P}\left(t_{0}-i \beta, t^{\prime}\right)$ fixes $A=$ $i f_{k}$. Therefore

$$
\mathcal{G}_{P}\left(t, t^{\prime}, \mathbf{k}\right)=-i\left(\Theta_{P}\left(t-t^{\prime}\right)-f_{k}\right) e^{-i \mathcal{E}_{k}\left(t-t^{\prime}\right)}
$$

The second equation 11 seems more complicated, but actually can be simplified by multiplying both sides by $i \sigma_{2}$ and using the identity $i \sigma_{2} \mathcal{D}^{-1}=\partial_{t}-\mathcal{M}$, where $\mathcal{M}$ satisfies $\mathcal{M}^{T}=-\sigma_{2} \mathcal{M} \sigma_{2}$. A simple computation gives

$$
\mathcal{D}_{P}\left(t, t^{\prime}\right)=e^{\mathcal{M}\left(t-t_{0}\right)} i \sigma_{2}\left[\Theta_{P}\left(t-t^{\prime}\right)+n\left(i \mathcal{M}^{T}\right)\right] e^{\mathcal{M}^{T}\left(t^{\prime}-t_{0}\right)}
$$

where the integration constant matrix $n(i \mathcal{M})$ is fixed by the boundary conditions $\mathcal{D}_{P}\left(t_{0}, t^{\prime}\right)=$ $\mathcal{D}_{P}\left(t_{0}-i \beta, t^{\prime}\right)$ to be

$$
n\left(i \mathcal{M}^{T}\right)=\frac{1}{\exp \left(i \beta \mathcal{M}^{T}\right)-1} .
$$

Notice that the time evolution is trivial and an explicit computation gives

$$
\mathcal{D}_{P}\left(t, t^{\prime}\right)=\left(\begin{array}{cc}
-\frac{c_{y y}}{\omega_{0}} \sin \omega_{0}\left(t-t^{\prime}\right) & \cos \omega_{0}\left(t-t^{\prime}\right) \\
-\cos \omega_{0}\left(t-t^{\prime}\right) & -\frac{c_{x x}}{\omega_{0}} \sin \omega_{0}\left(t-t^{\prime}\right)
\end{array}\right)\left[\Theta_{P}\left(t-t^{\prime}\right)+n\left(i \mathcal{M}^{T}\right)\right]
$$

Since $\mathcal{M}$ has eigenvalues $+i \omega_{0}$ and $-i \omega_{0}$, the eigenvalues of $n\left(i \mathcal{M}^{T}\right)$ are $n\left(-\omega_{0}\right)$ and $n\left(\omega_{0}\right)$ respectively, i.e., they are regular Bose-Einstein distributions. A little algebra allows to derive the free spectrum as

$$
<:\left\{S^{i}(t), S^{j}(t)\right\}:>=\left(\begin{array}{cc}
\frac{c_{y y}}{\omega_{0}} \frac{\sinh \beta \omega_{0}}{1-\cosh \beta \omega_{0}} & -i \\
i & \frac{c_{x x}}{\omega_{0}} \frac{\sinh \beta \omega_{0}}{1-\cosh \beta \omega_{0}}
\end{array}\right) .
$$

In the isotropic case, things are simpler since $\mathcal{M}$ is proportional to $i \sigma_{2}$ and therefore it commutes with $i \sigma_{2}$. We also observe that in the CTP formalism, each of the matrix equations 12 and 13, corresponds to an additional $2 \times 2$ matrix of equations in the Schwinger formalism, depending on the position of $t$ and $t^{\prime}$ in the path $P_{1}$ directed along the positive evolution in time and $P_{2}$ directed in the opposite direction. ${ }^{24}$ In particular 


$$
\begin{aligned}
& \mathcal{G}_{P}\left(t, t^{\prime}\right)=G_{\mathbf{k}}^{>}\left(t, t^{\prime}\right)=-i\left(1-f_{\mathbf{k}}\right) e^{-i \varepsilon_{\mathbf{k}}\left(t-t^{\prime}\right)}, t \in P_{2}, t^{\prime} \in P_{1} \\
& \mathcal{G}_{P}\left(t, t^{\prime}\right)=G_{\mathbf{k}}^{<}\left(t, t^{\prime}\right)=\quad i f_{\mathbf{k}} e^{-i \varepsilon_{\mathbf{k}}\left(t-t^{\prime}\right)}, \quad t \in P_{1}, t^{\prime} \in P_{2}
\end{aligned}
$$

It is easy to check that the previous propagators are consistent with the operator's expressions

$$
\begin{gathered}
G_{\mathbf{k}}^{>}\left(t, t^{\prime}\right)=-i<a_{\mathbf{k}}(t) a_{\mathbf{k}}^{\dagger}\left(t^{\prime}\right)>_{c}, \quad G_{\mathbf{k}}^{<}\left(t, t^{\prime}\right)=i<a_{\mathbf{k}}^{\dagger}\left(t^{\prime}\right) a_{\mathbf{k}}(t)>_{c} \\
D_{\mathbf{k}}^{i j>}\left(t, t^{\prime}\right)=-i<S_{-\mathbf{k}}^{i}(t) S_{\mathbf{k}}^{j}\left(t^{\prime}\right)>_{c}, \quad D_{\mathbf{k}}^{i j<}\left(t, t^{\prime}\right)=-i<S_{\mathbf{k}}^{j}\left(t^{\prime}\right) S_{-\mathbf{k}}^{i}(t)>_{c} .
\end{gathered}
$$

Next, we include the effect of the conduction electrons on the magnetization. Using the functional formulation, ${ }^{24}$ it is clear how to extract the effect of the electrons on the local magnetization field: it is enough to integrate out the fermionic degrees of freedom and to compute the effective action for the magnetization. Since the original action is quadratic in $\psi$ and $\psi^{\dagger}$, the functional integral can be performed. If we use a coherent state representation for the magnetization and the conduction electrons, the generating functional for the problem has the form ${ }^{25}$

$$
Z[J]=\int d z_{1} d z_{1}^{*} \int d \zeta_{1, i} d \zeta_{1, i}^{*} \exp \left[-z_{1} z_{1}^{*}-\zeta_{1, i} \zeta_{1, i}^{*}\right]\left\langle z_{1},-\zeta_{1}\left|\rho U^{\dagger}\left(J_{2}\right) U\left(J_{1}\right)\right| z_{1}, \zeta_{1}\right\rangle
$$

where $z$ 's represent the d-electrons degrees of freedom while $\zeta$ 's represent the conduction electrons. $J_{1}$ and $J_{2}$ are the usual virtual sources. The propagator inside is given by

$$
\begin{aligned}
\langle\|\|\rangle & =\int d z d z^{*} \int d \zeta d \zeta^{*} \exp \left[z_{1}^{*} z\left(t_{0}-i \beta\right)+\zeta_{1, k j}^{*} \zeta_{k j}\left(t_{0}-i \beta\right)\right. \\
& +i \int_{t_{0}}^{t_{0}-i \beta} d s\left(i z^{*} \partial_{s} z-H^{J}\left(z^{*}, z\right)\right) \\
& \left.+i \int_{t_{0}}^{t_{0}-i \beta} d s\left(i \zeta_{k j}^{*} \partial_{s} \zeta_{k j}+\zeta_{k j}^{*} \mathcal{G}_{k j j^{\prime}}^{-1} \zeta_{k j^{\prime}}\right)\right]
\end{aligned}
$$

where

$$
\mathcal{G}_{k j j^{\prime}}^{-1}(\mathbf{k}, \mathbf{p})=\left[\begin{array}{cc}
\left(i \partial_{s}-\varepsilon_{1}(\mathbf{k})\right) \delta(\mathbf{k}-\mathbf{p}) & \frac{J}{2} z(\mathbf{k}-\mathbf{p}) \\
\frac{J}{2} z^{*}(\mathbf{k}-\mathbf{p}) & \left(i \partial_{s}-\varepsilon_{2}(\mathbf{k})\right) \delta(\mathbf{k}-\mathbf{p})
\end{array}\right]
$$

the variable $s$ represents the time along the CTP $P$. In the above we have set the total number of magnetic cells $N=1$ since we the same discussion applies to a local atomic moment. Next we integrate out the conduction electrons degrees of freedom. Using 


$$
\int d \zeta^{*} d \zeta \exp \left[i \int d s \zeta^{*} \mathcal{G}^{-1} \zeta\right]=\exp \left[-\operatorname{Tr} \ln \mathcal{G}^{-1}\right]
$$

and expanding the logarithmic term gives exchange terms of all orders in the coupling constant $J$. In the following, we keep only quadratic terms. The conduction electron propagator satisfies the equation

$$
\left[\left(i \partial_{s}-\varepsilon_{i}(\mathbf{k})\right) \delta_{i j} \delta_{k, k^{\prime}}-V_{i j}\left(\mathbf{k}-\mathbf{k}^{\prime}\right)\right] \mathcal{G}_{j p}^{k k^{\prime}}\left(s-s^{\prime}\right)=-\delta_{i p}\left(s-s^{\prime}\right) \delta\left(\mathbf{k}-\mathbf{k}^{\prime}\right)
$$

with $V$, the interaction term is given by

$$
V\left(\mathbf{k}-\mathbf{k}^{\prime}\right)=\frac{J}{2}\left[\begin{array}{cc}
0 & z\left(\mathbf{k}-\mathbf{k}^{\prime}\right) \\
z^{*}\left(\mathbf{k}-\mathbf{k}^{\prime}\right) & 0
\end{array}\right] .
$$

This equation is solved by iteration, assuming that higher order terms are small. The large Zeeman-type term has been included in the $\varepsilon$ term. The propagator $\mathcal{G}$ is then given in terms of the propagator $\mathcal{G}^{(0)}$ which is a solution of the following equation

$$
\left(i \partial_{s}-\varepsilon_{i}(k)\right) \mathcal{G}_{i j}^{(0) k, k^{\prime}}\left(s, s^{\prime}\right)=-\delta_{i j}\left(s-s^{\prime}\right) \delta\left(\mathbf{k}-\mathbf{k}^{\prime}\right)
$$

If we define the functions $G^{<}$and $G^{>}$to be

$$
\begin{aligned}
& G^{<}\left(s, s^{\prime}\right)=-i f(\varepsilon) \exp \left[-i \varepsilon\left(s-s^{\prime}\right)\right], \\
& G^{>}\left(s, s^{\prime}\right)=i(1-f(\varepsilon)) \exp \left[-i \varepsilon\left(s-s^{\prime}\right)\right]
\end{aligned}
$$

then formally, we have

$$
\mathcal{G}^{(0)}\left(s, s^{\prime}\right)=G^{<}\left(s, s^{\prime}\right) \Theta_{P}\left(s^{\prime}-s\right)+G^{>}\left(s, s^{\prime}\right) \Theta_{P}\left(s-s^{\prime}\right),
$$

where $\Theta_{P}\left(s-s^{\prime}\right)$ is the step function along the closed-time path $P$. Hence, the presence of conduction electrons in the thin film will give rise to an effective action on the d-electrons. To order $J^{2}$, the logarithmic term becomes

$$
\begin{aligned}
\operatorname{Tr} \ln \mathcal{G} & =\frac{1}{2} \sum_{k, k^{\prime}} J^{2} \int d s d s^{\prime} \mathcal{G}_{i i, k k}^{(0)}\left(s, s^{\prime}\right) V_{i j, k k^{\prime}}\left(s^{\prime}\right) \mathcal{G}_{j j, k^{\prime} k^{\prime}}^{(0)}\left(s^{\prime}, s\right) V_{j i, k^{\prime} k}(s) \\
& =J^{2} \sum_{k, k^{\prime}} \int_{c} d s d s^{\prime} z\left(s^{\prime}, \mathbf{k}-\mathbf{k}^{\prime}\right) z^{*}\left(s, \mathbf{k}^{\prime}-\mathbf{k}\right) \exp \left[-i\left(\varepsilon_{1}(\mathbf{k})-\varepsilon_{2}\left(\mathbf{k}^{\prime}\right)\right)\left(s-s^{\prime}\right)\right] \\
& \times\left[\Theta\left(s-s^{\prime}\right) f\left(\varepsilon_{2}\left(\mathbf{k}^{\prime}\right)\right)\left(1-f\left(\varepsilon_{1}(\mathbf{k})\right)\right)+\Theta\left(s^{\prime}-s\right) f\left(\varepsilon_{1}(\mathbf{k})\right)\left(1-f\left(\varepsilon_{2}\left(\mathbf{k}^{\prime}\right)\right)\right)\right]
\end{aligned}
$$


which can be written in a more compact form as follows

$$
\begin{aligned}
\operatorname{Tr} \ln \mathcal{G} & =J^{2} \sum_{k, k^{\prime}} \int_{t_{0}}^{t} d t d t^{\prime} \exp \left[-i\left(\varepsilon_{1}(\mathbf{k})-\varepsilon_{2}\left(\mathbf{k}^{\prime}\right)\right)\left(t-t^{\prime}\right)\right] \\
& \times z_{i}^{*}\left(t, \mathbf{k}^{\prime}-\mathbf{k}\right) \mathfrak{A}_{i j}\left(t-t^{\prime}, \mathbf{k}, \mathbf{k}^{\prime}\right) z_{j}\left(t^{\prime}, \mathbf{k}-\mathbf{k}^{\prime}\right)
\end{aligned}
$$

In matrix form,

$$
\begin{aligned}
\mathfrak{A}_{11}\left(t-t^{\prime}, \mathbf{k}, \mathbf{k}^{\prime}\right)= & \Theta\left(t-t^{\prime}\right) f\left(\varepsilon_{2}\left(\mathbf{k}^{\prime}\right)\right)\left(1-f\left(\varepsilon_{1}(\mathbf{k})\right)\right) \\
& +\Theta\left(t^{\prime}-t\right) f\left(\varepsilon_{1}(\mathbf{k})\right)\left(1-f\left(\varepsilon_{2}\left(\mathbf{k}^{\prime}\right)\right)\right), \\
\mathfrak{A}_{12}\left(t-t^{\prime}, \mathbf{k}, \mathbf{k}^{\prime}\right)= & f\left(\varepsilon_{1}(\mathbf{k})\right)\left(1-f\left(\varepsilon_{2}\left(\mathbf{k}^{\prime}\right)\right)\right) \\
\mathfrak{A}_{21}\left(t-t^{\prime}, \mathbf{k}, \mathbf{k}^{\prime}\right)= & f\left(\varepsilon_{2}\left(\mathbf{k}^{\prime}\right)\right)\left(1-f\left(\varepsilon_{1}(\mathbf{k})\right)\right) \\
\mathfrak{A}_{22}\left(t-t^{\prime}, \mathbf{k}, \mathbf{k}^{\prime}\right)= & \Theta\left(t^{\prime}-t\right) f\left(\varepsilon_{2}\left(\mathbf{k}^{\prime}\right)\right)\left(1-f\left(\varepsilon_{1}(\mathbf{k})\right)\right) \\
& +\Theta\left(t-t^{\prime}\right) f\left(\varepsilon_{1}(\mathbf{k})\right)\left(1-f\left(\varepsilon_{2}\left(\mathbf{k}^{\prime}\right)\right)\right) .
\end{aligned}
$$

where $f\left(\varepsilon_{\sigma}\right)$ is the Fermi-Dirac distribution for spin-up $(\sigma=1)$ and $\operatorname{spin}$ down $(\sigma=2)$, respectively. The effective action of the magnetization is now given by

$$
\begin{aligned}
S^{e f f} & =\int d t\left[i z_{1}^{*} \partial_{t} z_{1}-H^{J}\left(z_{1}^{*}, z_{1}\right)\right] \\
& -\int d t\left[i z_{2}^{*} \partial_{t} z_{2}-H^{J}\left(z_{2}^{*}, z_{2}\right)\right]+i \operatorname{Tr} \ln \mathcal{G}
\end{aligned}
$$

where $z=S_{+}=S_{x} \pm i S_{y}$. Next, we make a change of variables

$$
\left(\begin{array}{c}
z_{1} \\
z_{2}
\end{array}\right)=\left(\begin{array}{cc}
1 & 1 / 2 \\
1 & -1 / 2
\end{array}\right)\left(\begin{array}{l}
\mathfrak{Z} \\
\mathfrak{z}
\end{array}\right) \equiv T\left(\begin{array}{l}
\mathfrak{Z} \\
\mathfrak{z}
\end{array}\right)
$$

The new quadratic form is then

$$
\sum_{i j} z_{i}^{*} \mathfrak{A}_{i j} z_{j}=\left(\mathfrak{Z}^{*} \mathfrak{z}^{*}\right) T^{T} \mathfrak{A} T\left(\begin{array}{l}
\mathfrak{Z} \\
\mathfrak{z}
\end{array}\right) .
$$

This form first appeared in Schwinger's paper ${ }^{24}$ but is often cited under the name of the 
Keldysh form ${ }^{26}$,

$$
\begin{aligned}
\mathcal{U}\left(t-t^{\prime}, \mathbf{k}, \mathbf{k}^{\prime}\right) & =T^{T} \mathfrak{A} T \\
\mathcal{U}_{11}\left(t-t^{\prime}, \mathbf{k}, \mathbf{k}^{\prime}\right) & =0 \\
\mathcal{U}_{12}\left(t-t^{\prime}, \mathbf{k}, \mathbf{k}^{\prime}\right) & =\Theta\left(t^{\prime}-t\right)\left[f\left(\varepsilon_{1}(\mathbf{k})\right)-f\left(\varepsilon_{2}\left(\mathbf{k}^{\prime}\right)\right)\right], \\
\mathcal{U}_{21}\left(t-t^{\prime}, \mathbf{k}, \mathbf{k}^{\prime}\right) & =-\Theta\left(t-t^{\prime}\right)\left[f\left(\varepsilon_{1}(\mathbf{k})\right)-f\left(\varepsilon_{2}\left(\mathbf{k}^{\prime}\right)\right)\right], \\
\mathcal{U}_{22}\left(t-t^{\prime}, \mathbf{k}, \mathbf{k}^{\prime}\right) & =\frac{1}{2} f\left(\varepsilon_{1}(\mathbf{k})\right)\left(1-f\left(\varepsilon_{2}\left(\mathbf{k}^{\prime}\right)\right)\right)+\frac{1}{2} f\left(\varepsilon_{2}\left(\mathbf{k}^{\prime}\right)\right)\left(1-f\left(\varepsilon_{1}(\mathbf{k})\right)\right) .
\end{aligned}
$$

It is easy to see from these definitions of the kernels $\mathcal{U}_{i j}$ that

$$
\begin{aligned}
& \mathcal{U}_{12}\left(\mathbf{k}, \mathbf{k}^{\prime}, t\right)=-\mathcal{U}_{21}\left(\mathbf{k}, \mathbf{k}^{\prime},-t\right) \\
& \mathcal{U}_{22}\left(\mathbf{k}, \mathbf{k}^{\prime}, t\right)=\mathcal{U}_{22}\left(\mathbf{k}, \mathbf{k}^{\prime},-t\right)
\end{aligned}
$$

We can now introduce a new field $\eta$ into the theory. This will mimic a random Gaussian field in the semi-classical equations. In the generating functional $Z[J]$, we replace the quadratic term in $\mathfrak{z}$ and $\mathfrak{z}^{*}$ by linear terms,

$$
\begin{aligned}
& \exp \left[-\left(\frac{J}{2}\right)^{2} \sum_{k k^{\prime}} \int d t \int d t^{\prime} \mathfrak{z}^{*}\left(t, \mathbf{k}^{\prime}-\mathbf{k}\right) \mathcal{U}_{22}\left(t-t^{\prime}, \mathbf{k}, \mathbf{k}^{\prime}\right)\right. \\
& \left.\exp \left[-i\left(\varepsilon_{1}(\mathbf{k})-\varepsilon_{2}\left(\mathbf{k}^{\prime}\right)\right)\left(t-t^{\prime}\right)\right] \mathfrak{z}\left(t^{\prime}, \mathbf{k}-\mathbf{k}^{\prime}\right)\right] \\
& =\int d \eta^{*} d \eta \exp \left[-\sum_{k k^{\prime}} \int d t \int d t^{\prime} \eta^{*}(t, \mathbf{k}) \mathfrak{D}^{-1}\left(t, \mathbf{k} ; t^{\prime}, k^{\prime}\right) \eta\left(t^{\prime}, \mathbf{k}^{\prime}\right)\right. \\
& \left.+i \sum_{k} \int d t \eta^{*}(t, \mathbf{k}) \mathfrak{z}(t, \mathbf{k})+\mathfrak{z}^{*}(t, \mathbf{k}) \eta(t, \mathbf{k})\right]
\end{aligned}
$$

where the kernel $\mathfrak{D}$ is given by

$$
\begin{aligned}
\mathfrak{D}\left(t, \mathbf{k} ; t^{\prime}, \mathbf{k}^{\prime}\right)= & \frac{J^{2}}{2} \exp \left[-i\left(\varepsilon_{1}(\mathbf{k})-\varepsilon_{2}\left(\mathbf{k}^{\prime}\right)\right)\left(t-t^{\prime}\right)\right] \\
& \times\left(f\left(\varepsilon_{1}(\mathbf{k})\right)-f\left(\varepsilon_{2}\left(\mathbf{k}^{\prime}\right)\right)\right) \operatorname{coth}\left[\frac{\beta\left(\varepsilon_{1}(\mathbf{k})-\varepsilon_{2}\left(\mathbf{k}^{\prime}\right)\right)}{2}\right] .
\end{aligned}
$$

In the bulk, this noise kernel will in general depend on the relaxation of the conduction electrons due to phonons which will appear in the exponential term on the right hand side. 
After this integral transformation, the effective action becomes

$$
\begin{aligned}
i S^{e f f} & =-\int d t\left\{\frac{1}{2} \mathfrak{Z}^{*} \partial_{t \mathfrak{z}}+\frac{1}{2} \mathfrak{z}^{*} \partial_{t} \mathfrak{Z}-\frac{1}{2} \mathfrak{Z} \partial_{t} \mathfrak{z}^{*}-\frac{1}{2} \mathfrak{z} \partial_{t} \mathfrak{Z}^{*} \mid\right. \\
& \left.-i \Omega\left(\mathfrak{Z}^{*} \mathfrak{z}+\mathfrak{z}^{*} \mathfrak{Z}\right)-i K\left(\mathfrak{Z}^{*} \mathfrak{z}^{*}+\mathfrak{z} \mathfrak{Z}\right)\right\} \\
& +\left(\frac{J}{2}\right)^{2} \sum_{k k^{\prime}} \int d t \int d t^{\prime} \exp \left[-i\left(\varepsilon_{1}(\mathbf{k})-\varepsilon_{2}\left(\mathbf{k}^{\prime}\right)\right)\left(t-t^{\prime}\right)\right] \\
& \times\left\{\mathfrak{Z}^{*}\left(t, \mathbf{k}^{\prime}-\mathbf{k}\right) \mathcal{U}_{12}\left(t-t^{\prime}, \mathbf{k}, \mathbf{k}^{\prime}\right) \mathfrak{z}\left(t^{\prime}, \mathbf{k}-\mathbf{k}^{\prime}\right)\right. \\
& \left.+\mathfrak{z}\left(t, \mathbf{k}^{\prime}-\mathbf{k}\right) \mathcal{U}_{21}\left(t-t^{\prime}, \mathbf{k}, \mathbf{k}^{\prime}\right) \mathfrak{Z}\left(t^{\prime}, \mathbf{k}-\mathbf{k}^{\prime}\right)\right\} \\
& +i \sum_{k} \int d t\left\{\eta^{*}(t, \mathbf{k}) \mathfrak{z}(t, \mathbf{k})+\mathfrak{z}^{*}(t, \mathbf{k}) \eta(t, \mathbf{k})\right\}+F\left[\eta^{*}, \eta\right] .
\end{aligned}
$$

The equations of motion are obtained by minimizing the action with respect to the variables $\mathfrak{z}$ and $\mathfrak{z}^{*}$,

and

$$
\left.\frac{\delta\left(S^{\text {eff }}\right)}{\delta \mathfrak{z}(t, \mathbf{p})}\right|_{\eta^{*}=\eta=0}=0
$$

$$
\eta(t, \mathbf{x})=\eta_{x}(t, \mathbf{x})+i \eta_{y}(t, \mathbf{x})
$$

We find that the transverse components of the local magnetization satisfy the following equations of motion

$$
\begin{aligned}
\frac{d S_{x}(t, \mathbf{p})}{d t} & =(\Omega-K) S_{y}(t, \mathbf{p})-\eta_{y}(t, \mathbf{p}) \\
& +\left(\frac{J}{2}\right)^{2} \sum_{\mathbf{k}} \int d t^{\prime} \mathcal{K}\left(t, \mathbf{k} ; t^{\prime}, \mathbf{k}+\mathbf{p}\right) \cos \left[\left(\varepsilon_{1}(\mathbf{k})-\varepsilon_{2}(\mathbf{k}+\mathbf{p})\right)\left(t-t^{\prime}\right)\right] S_{x}\left(t^{\prime},-\mathbf{p}\right) \\
& +\left(\frac{J}{2}\right)^{2} \sum_{\mathbf{k}} \int d t^{\prime} \mathcal{K}\left(t, \mathbf{k} ; t^{\prime}, \mathbf{k}+\mathbf{p}\right) \sin \left[\left(\varepsilon_{1}(\mathbf{k})-\varepsilon_{2}(\mathbf{k}+\mathbf{p})\right)\left(t-t^{\prime}\right)\right] S_{y}\left(t^{\prime},-\mathbf{p}\right),
\end{aligned}
$$

and

$$
\begin{aligned}
\frac{d S_{y}(t, \mathbf{p})}{d t} & =-(\Omega+K) S_{y}(t, \mathbf{p})+\eta_{x}(t, \mathbf{p}) \\
& -\left(\frac{J}{2}\right)^{2} \sum_{\mathbf{k}} \int d t^{\prime} \mathcal{K}\left(t, \mathbf{k} ; t^{\prime}, \mathbf{k}+\mathbf{p}\right) \sin \left[\left(\varepsilon_{1}(\mathbf{k})-\varepsilon_{2}(\mathbf{k}+\mathbf{p})\right)\left(t-t^{\prime}\right)\right] S_{x}\left(t^{\prime},-\mathbf{p}\right) \\
& +\left(\frac{J}{2}\right)^{2} \sum_{\mathbf{k}} \int d t^{\prime} \mathcal{K}\left(t, \mathbf{k} ; t^{\prime}, \mathbf{k}+\mathbf{p}\right) \cos \left[\left(\varepsilon_{1}(\mathbf{k})-\varepsilon_{2}(\mathbf{k}+\mathbf{p})\right)\left(t-t^{\prime}\right)\right] S_{y}\left(t^{\prime},-\mathbf{p}\right)
\end{aligned}
$$


where

$$
\mathcal{K}\left(t, \mathbf{k} ; t^{\prime}, \mathbf{k}+\mathbf{p}\right)=\Theta\left(t-t^{\prime}\right)\left[f\left(\varepsilon_{1}(\mathbf{k})\right)-f\left(\varepsilon_{2}(\mathbf{k}+\mathbf{p})\right)\right]
$$

and the correlation function for the random field due to the conduction electrons is given by

$$
\begin{aligned}
\left\langle\eta^{*}(t, \mathbf{p}) \eta\left(t^{\prime}, \mathbf{p}^{\prime}\right)\right\rangle & =\frac{J^{2}}{2}\left(f\left(\varepsilon_{1}(\mathbf{p})\right)-f\left(\varepsilon_{2}\left(\mathbf{p}^{\prime}\right)\right)\right) \operatorname{coth}\left[\frac{\beta}{2}\left(\varepsilon_{2}\left(\mathbf{p}^{\prime}\right)-\varepsilon_{1}(\mathbf{p})\right)\right] \\
& \times \exp \left[-i\left(\varepsilon_{1}(\mathbf{p})-\varepsilon_{2}\left(\mathbf{p}^{\prime}\right)\right)\left(t-t^{\prime}\right)\right] .
\end{aligned}
$$

The correlation function of the $\mathrm{x}$-component is then given by

$\operatorname{Re}\left\langle\eta_{x}(t,-\mathbf{k}) \eta_{x}\left(t^{\prime}, \mathbf{k}^{\prime}\right)\right\rangle=\left(\frac{J}{2}\right)^{2}\left(f\left(\varepsilon_{1}(\mathbf{k})\right)-f\left(\varepsilon_{2}\left(\mathbf{k}^{\prime}\right)\right)\right) \operatorname{coth}\left[\frac{\beta \omega_{k, k^{\prime}}}{2}\right] \cos \left[\omega_{k, k^{\prime}}\left(t-t^{\prime}\right)\right]$,

where $\omega_{k, k^{\prime}}=\varepsilon_{1}(\mathbf{k})-\varepsilon_{2}\left(\mathbf{k}^{\prime}\right)$. This is one of the important results in this work. The kernel terms account for the dissipation and a shift in the frequency due to the interaction with the conduction electrons. These equations of motion differ from the usual Gilbert form since the dissipation term is not a derivative and is non-local. These equations generalize those derived by Mills for a Stoner particle at zero temperature and in the adiabatic limit. ${ }^{9}$ Hence the memory terms will be very important for local moments. In the next section we study the limit under which the GB equation is recovered in a thin film embedded between two large reservoirs at equilibrium.

\section{FINITE-SIZE EFFECTS IN THE MEMORYLESS LIMIT}

In a series of very illuminating papers, Simanek was able to show how the ideas of Tserkovniak, Brataas and Bauer can be understood in the familiar linear response approach which avoids the use of the scattering method. ${ }^{6,8,10}$ Similar calculations were carried out by Mills ${ }^{9}$ using a dynamic RKKY approach which generalizes the earlier results obtained by Berger $^{5}$. It is well known that the spin-pumping theory and the Berger-Mills theory both give interfacial additional damping due to spin currents (not charge currents). In the former theory, this damping vanishes when there is no exchange splitting between the spin-up and spin-down electrons while it does not in the Berger-Mills theory. The spin pumping theory however seems to be very successful in interpreting the recent experiments by Mizukami et al. ${ }^{27}$. Hence in this section, we use the equations derived in the previous section to further understand this particular discrepancy between the various methods. Our results happen 
to be similar to those derived by Simanek using the spin-pumping theory. We believe however that our approach is more direct and transparent besides it is self-contained. This equivalence has very important consequences on the understanding of the physical origin of the spin momentum torque in finite films. Moreover, our theory is easy to extend to finite temperature and can deal with transient conditions as we show in the next section.

First, let's set-up the geometry of the problem and calculate the damping in the limit when there is no memory in the magnetic system, i.e., the average magnetization is much slower than the conduction electrons. This is the adiabatic limit. The geometry we adopt (fig. 4 is the same as the one adopted by $\mathrm{TBB}^{6}$. The two reservoirs on each side of the thin film will act as a sink for the spin leaked through the interfaces. The reservoirs are maintained at the same chemical potential $\mu$ in this section and hence there is no net flow of charge from left to right. Our theory can be also adapted to the case of non-equal chemical potentials which is briefly addressed in the following section.

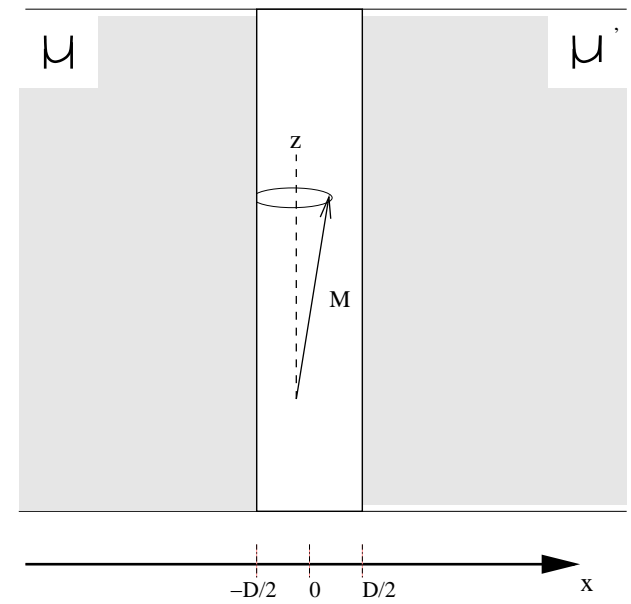

FIG. 4: A thin film confined between two large reservoirs with the same chemical potentials $\mu=\mu^{\prime}$. The case of different $\mu$ 's is treated in sect. IV.

For the rest of this section we use Mills ${ }^{9}$ notation since he was able to derive a more general form for the Gilbert equation in the adiabatic limit. We will show below how our theory reduces to his in this limit.

Within the linear response approach, the Gilbert equation for the magnetization $\mathbf{M}$ is

$$
\begin{aligned}
\frac{\partial \mathbf{M}}{\partial t}= & -|\gamma|\left[\mathbf{M} \times\left(\mathbf{H}+\left\langle\mathbf{H}_{e f f}(t)\right\rangle\right)\right] \\
& +\frac{G|\gamma|}{\gamma^{2} M_{s}^{2}}\left[\mathbf{M} \times \frac{\partial \mathbf{M}}{\partial t}\right]
\end{aligned}
$$


where $G$ is the Gilbert constant and the effective field $\mathbf{H}_{\text {eff }}$ is due to the action of the conduction electrons with the magnetization through a sd-type interaction

$$
\begin{aligned}
& H_{x}^{e f f}(t)=\frac{J^{2} V_{c}}{2 N \hbar \mu_{0}^{2}}\left(\wedge_{2} \frac{d M_{x}}{d t}+\wedge_{1} \frac{d M_{y}}{d t}\right), \\
& H_{y}^{e f f}(t)=\frac{J^{2} V_{c}}{2 N \hbar \mu_{0}^{2}}\left(\wedge_{2} \frac{d M_{y}}{d t}-\wedge_{1} \frac{d M_{x}}{d t}\right) .
\end{aligned}
$$

Hence the conduction electrons enhance the Gilbert damping term by

$$
\Delta G=\frac{J^{2} V_{c}}{2 N \hbar \mu_{0}^{2}} \wedge_{2}|\gamma|^{2} M_{s}^{2} .
$$

It was moreover argued by Mills that the constant $\wedge_{1}$, which renormalizes the precessional frequency, is not zero in general as was assumed by Simanek and Heinrich ${ }^{8}$. Below we show that our analysis naturally gives an explicit expression for this term and that it vanishes within the approximations employed here. In higher orders in $J^{2}$, the contribution of this term is non-zero but small as we show in the next section.

From the equations of motion for the x,y-components, Eqs. 50-51, we easily see that it is the term that has the sine-dependence that gives rise to damping,

$$
\begin{aligned}
\Delta G_{x(y)}(t, \mathbf{p})= & \frac{J^{2}}{2} \int_{0}^{\infty} d t^{\prime} \sum_{k}\left[f\left(\varepsilon_{1}(\mathbf{k})-f\left(\varepsilon_{2}(\mathbf{k}+\mathbf{p})\right)\right]\right. \\
& \times \sin \left[\left(\varepsilon_{1}(\mathbf{k})-\varepsilon_{2}(\mathbf{k}+\mathbf{p})\right) t^{\prime}\right] S_{y(x)}\left(t-t^{\prime},-\mathbf{p}\right) .
\end{aligned}
$$

The cosine-dependent term will be shown below to be the origin of the term $\wedge_{1}$ discussed by Mills. If we use the slow (adiabatic) precession approximation for the spin variables,

$$
S_{x(y)}\left(t-t^{\prime},-\mathbf{p}\right)=S_{x(y)}(t,-\mathbf{p})-t^{\prime} \frac{d S_{x(y)}(t,-\mathbf{p})}{d t}+\ldots
$$

Inserting this back in Eq. 58, we get

$$
\Delta G_{x(y)}(t, \mathbf{p})=\Delta G_{x(y)}^{(p)}(t, \mathbf{p})+\Delta G_{x(y)}^{(d)}(t, \mathbf{p}),
$$

with

$$
\begin{aligned}
\Delta G_{x(y)}^{(p)}(t, \mathbf{p})= & \frac{J^{2}}{2} S_{y(x)}(t,-\mathbf{p}) \sum_{\mathbf{k}} \int_{0}^{\infty} d t^{\prime}\left[f\left(\varepsilon_{1}(\mathbf{k})-f\left(\varepsilon_{2}(\mathbf{k}+\mathbf{p})\right)\right]\right. \\
& \times \sin \left[\left(\varepsilon_{1}(\mathbf{k})-\varepsilon_{2}(\mathbf{k}+\mathbf{p})\right) t^{\prime}\right]
\end{aligned}
$$


and

$$
\begin{aligned}
\Delta G_{x(y)}^{(d)}(t, \mathbf{p})= & -\frac{J^{2}}{2} \frac{d S_{y(x)}(t,-\mathbf{p})}{d t} \sum_{\mathbf{k}} \int_{0}^{\infty} d t^{\prime}\left[f\left(\varepsilon_{1}(\mathbf{k})-f\left(\varepsilon_{2}(\mathbf{k}+\mathbf{p})\right)\right]\right. \\
& \times t^{\prime} \sin \left[\left(\varepsilon_{1}(\mathbf{k})-\varepsilon_{2}(\mathbf{k}+\mathbf{p})\right) t^{\prime}\right]
\end{aligned}
$$

The term $\Delta G_{x(y)}^{(p)}$ contributes to the precessional frequency while $\Delta G_{x(y)}^{(d)}$ gives rise to Gilbert type damping. Hence it should be related to the imaginary part of a susceptibility term. In fact we can write

$$
\Delta G_{x(y)}^{(d)}(t, \mathbf{p})=-\left.i \frac{d \chi(\Omega, \mathbf{p})}{d \Omega}\right|_{\Omega=0} \frac{d S_{y(x)}(t,-\mathbf{p})}{d t},
$$

where the 'susceptibility' function $\chi$ is

$$
\chi(t, \mathbf{p})=-\frac{J^{2}}{2} \Theta(t) \sum_{\mathbf{k}}\left[f\left(\varepsilon_{1}(\mathbf{k})\right)-f\left(\varepsilon_{2}(\mathbf{k}+\mathbf{p})\right)\right] \sin \left[t\left(\varepsilon_{1}(\mathbf{k})-\varepsilon_{2}(\mathbf{k}+\mathbf{p})\right)\right],
$$

and its Fourier transform

$$
\begin{aligned}
\chi(\Omega, \mathbf{p})= & \int_{-\infty}^{\infty} \chi(t, \mathbf{p}) e^{i \Omega t} d t \\
= & -\frac{J^{2}}{4} \sum_{\mathbf{k}}\left[f\left(\varepsilon_{1}(\mathbf{k})-f\left(\varepsilon_{2}(\mathbf{k}+\mathbf{p})\right)\right]\right. \\
& \times\left[\frac{1}{\varepsilon_{1}(\mathbf{k})-\varepsilon_{2}(\mathbf{k}+\mathbf{p})+\Omega+i \eta}+\frac{1}{\varepsilon_{1}(\mathbf{k})-\varepsilon_{2}(\mathbf{k}+\mathbf{p})-\Omega-i \eta}\right],
\end{aligned}
$$

where $\eta$ is a small positive real number. From this expression, we see that to allow for a finite relaxation time $\tau_{s}$ of the conduction electrons, we can just replace $\Omega$ by $\Omega+i / \tau_{s}$. The constants $\Lambda_{2}$ introduced by Mills can be obtained from $\chi$ through the following expression

$$
-\left.i \frac{d \chi(\Omega, \mathbf{p})}{d \Omega}\right|_{\Omega=0}=\Lambda_{2 r}(\mathbf{p})+i \Lambda_{2}(\mathbf{p})
$$

An easy calculation shows that $\Lambda_{2 r}$ vanishes within this approximation and $\Lambda_{2}$, which is still a function of the momentum, is given by

$$
\begin{aligned}
\Lambda_{2}(\mathbf{p})= & -J^{2} \sum_{\mathbf{k}}\left[f\left(\varepsilon_{1}(\mathbf{k})\right)-f\left(\varepsilon_{2}(\mathbf{k}+\mathbf{p})\right)\right]\left(\varepsilon_{1}(\mathbf{k})-\varepsilon_{2}(\mathbf{k}+\mathbf{p})\right) \\
& \times\left[\frac{\eta}{\left(\left(\varepsilon_{1}(\mathbf{k})-\varepsilon_{2}(\mathbf{k}+\mathbf{p})\right)^{2}+\eta^{2}\right)^{2}}\right]
\end{aligned}
$$

As shown by Heinrich, Fraitova and Kambersky ${ }^{15}$ in an infinite medium, the conduction electrons can't dissipate energy unless the electron-hole pairs have a finite-lifetime by transferring energy to the lattice. This can be done by taking a temperature-dependent finite 
$\eta$. In this case the damping term $\Lambda_{2}(\mathbf{p})$ will not vanish. This spin-flipping mechanism is believed to be the source of the damping in iron and permalloy ${ }^{28}$. Another source for damping can be geometrical in origin. As shown by Mills a breakdown of wave vector conservation due of the finite size of the film can give rise to dissipation. In the language of reservoirs, we rephrase this by saying that quenching the states of the magnetic film to a countable number while leaving those of the electronic states denumerable is equivalent to a Caldeira-Leggett (CL) model with a fermionic bath which is known to give rise to quantum dissipation. $^{4,29}$ Our geometry is then a typical example of this model and should show a dissipative behavior as a function of the thickness $D$, i.e., $\Lambda_{2}(p) \rightarrow 0$ as $D \rightarrow \infty$, even in the case of very slow relaxation times for the conduction electrons. We take the thin film to have finite thickness $D$ in the x-direction, and we take the transverse components, $S_{x}$ and $S_{y}$ to dependent only on the x-coordinate normal to the plane with pinned or unpinned boundary conditions. In the continuum approximation, the magnetization components in this symmetric configuration take the form

$$
S_{i}(t, \mathbf{r})=\sum_{n=0,1,2, \ldots} S_{i}^{n}(t) \cos \left(\frac{n \pi}{D} x\right), \quad i=x, y
$$

The Fourier transform is given by

$$
\int d x S_{i}(t, x) e^{i p x}=S_{i}(t, p)
$$

therefore, we have $S_{i}(t,-p)=S_{i}^{*}(t, p)$ and

$$
S_{i}(t, \mathbf{p})=(2 \pi)^{2} \delta\left(\mathbf{p}_{\|}\right) \sum_{n} S_{i}^{n}(t)\left[\frac{\sin \left(\left(p+\frac{n \pi}{D}\right) D / 2\right)}{\left(p+\frac{n \pi}{D}\right)}+\frac{\sin \left(\left(p-\frac{n \pi}{D}\right) D / 2\right)}{\left(p-\frac{n \pi}{D}\right)}\right],
$$

where we have set $p_{x}=p,-\infty<p<\infty$.

In the following we assume that the splitting $\delta$ in electronic energy bands due to the sd-interaction is smaller than the Fermi energy and $k_{B} T<<\mu$ which is the case at room temperature, then it is enough for our purposes (because of the finite size) to use the following approximation for the Fermi-Dirac functions for the conduction electrons

$$
\begin{aligned}
{\left[f\left(\varepsilon_{2}(\mathbf{k}+\mathbf{p})\right)-f\left(\varepsilon_{1}(\mathbf{k})\right]\right.} & \cong \frac{\partial f}{\partial \varepsilon_{\mathbf{k}}}\left(\varepsilon_{2}(\mathbf{k}+\mathbf{p})-\varepsilon_{1}(\mathbf{k})\right) \\
& =\left.\frac{\partial f}{\partial \varepsilon_{\mathbf{k}}}\right|_{\bar{\varepsilon}_{F}}\left(\frac{\mathbf{k} \cdot \mathbf{p}}{m}+\delta\right)
\end{aligned}
$$

where $\bar{\varepsilon}_{F}=\left(\varepsilon_{F}^{\uparrow}+\varepsilon_{F}^{\downarrow}\right) / 2$. This approximation is not necessary and will not change our conclusions but it helps keep the algebra at minimum, otherwise the Lindhard function 
will appear explicitly in our expressions and will considerably add to the complexity of the calculations. If a non-zero voltage difference is applied across the thin film, then Eq. 71 has to be modified to take account of the spin accumulation effect $\Delta^{\uparrow \downarrow}=\mu^{\uparrow}-\mu^{\downarrow}$ due to the normal-ferromagnetic interface ${ }^{30}$. This term which can be positive or negative depending on the direction of the polarized current will hence contribute to the damping. We will say more about this case when we study the noise and the corresponding fluctuation dissipation theorem in the next section. Using the approximation Eq. 71, the damping term becomes $($ for $p \neq 0)$

$$
\begin{aligned}
\wedge_{2}(p)= & \frac{J^{2} v}{2(2 \pi)^{2} \tau_{s}} \frac{m}{p \varepsilon_{F}}\left[\frac{\delta-p \sqrt{\frac{2 \varepsilon_{F}}{m}}}{\left[\left(\delta-p \sqrt{\frac{2 \varepsilon_{F}}{m}}\right)^{2}+\tau_{s}^{-2}\right]}-\frac{\delta+p \sqrt{\frac{2 \varepsilon_{F}}{m}}}{\left[\left(\delta+p \sqrt{\frac{2 \varepsilon_{F}}{m}}\right)^{2}+\tau_{s}^{-2}\right]}\right. \\
& \left.-\tau_{s}\left(\tan ^{-1}\left[\tau_{s}\left(\delta-p \sqrt{\frac{2 \varepsilon_{F}}{m}}\right)\right]-\tan ^{-1}\left[\tau_{s}\left(\delta+p \sqrt{\frac{2 \varepsilon_{F}}{m}}\right)\right]\right)\right],
\end{aligned}
$$

where the Fermi energy $\varepsilon_{F}$ is that of the spin up electron in the ferromagnet. Care is needed to get the corresponding expression for $p=0$. The damping is therefore momentumdependent as we should expect in a finite film. This expression can be, e.g., useful for studies of spin-wave resonance in thin films. In the remaining, we confine ourselves to the volume mode since it is usually the mode measured by FMR. In this case, the damping simply becomes

$$
\bar{\wedge}_{2} \simeq \frac{4}{\pi D} \int_{0}^{\infty} d p\left(\frac{\sin \left(p \frac{D}{2}\right)}{p}\right)^{2} \wedge_{2}(p) .
$$

As it is clear from this expression, damping is directly related to the breakdown of momentum conservation in the direction normal to the film. This damping clearly vanishes when the size of the film becomes infinite, i.e., $D \rightarrow \infty$. This expression for the damping was found by Fourier transforming back the equations of motion, eqs. 50-51, to real-space and enforce the condition that the magnetization vanishes for $|x|>D / 2$. This is the procedure that we followed to allow us to capture the finite size effects of the magnetic film on the damping in this section and on the noise in the next one. Using the fact that the saturation magnetization is defined by $M_{s}=\mu_{0} / v$, the excess Gilbert damping is therefore given by

$$
G_{s}=\frac{\mu_{0}^{2}}{v} \bar{\wedge}_{2}
$$

We write this in terms of small dimensionless parameters $\delta_{r}=\frac{\delta}{\varepsilon_{F}}$ and $\tau_{r}=\frac{1}{\tau_{s}} / \varepsilon_{F}$ with 
typical values 0.4 and 0.001 , respectively in transition metals. The damping is now given by

$$
G_{s}=\delta_{r}^{2} \frac{\mu_{0}^{2} k_{F}^{2}}{2 \pi^{2} \hbar D} F\left(\tau_{r}, \delta_{r}, D\right),
$$

with

$$
\begin{aligned}
F\left(\tau_{r}, \delta_{r}, D\right) & =\frac{\tau_{r}}{\pi} \int_{0}^{\infty} d x \frac{1}{x^{2}} \sin ^{2}\left(\frac{1}{4} k_{F} D x\right)\left\{\frac{\delta_{r}-x}{x\left[\left(\delta_{r}-x\right)^{2}+\tau_{r}^{2}\right]}\right. \\
& -\frac{\delta_{r}+x}{x\left[\left(\delta_{r}+x\right)^{2}+\tau_{r}^{2}\right]}-\frac{\tan ^{-1}\left[\left(\delta_{r}-x\right) / \tau_{r}\right]}{x \tau_{r}} \\
& \left.+\frac{\tan ^{-1}\left[\left(\delta_{r}+x\right) / \tau_{r}\right]}{x \tau_{r}}\right\}
\end{aligned}
$$

In the limit of vanishing bulk damping, $\tau_{r} \rightarrow 0$, and large thickness $D$, the damping is given by

$$
G_{s}=\frac{\mu_{0}^{2} k_{F}^{2}}{2 \pi^{2} \hbar}\left(\frac{0.55 \delta_{r}}{D}\right) .
$$

To get this result, we used the approximation

$$
f(x)=\int_{x}^{\infty} d y \frac{\sin ^{2} y}{y^{2}} \approx \frac{0.55}{x},
$$

for large $x$. Hence this damping is due to finite-size effects. At smaller thicknesses, the damping shows some oscillatory behavior as shown in figs 5,6,7 for small bulk damping. The size $\left(\approx 10^{8} \sec ^{-1}\right)$ and the oscillatory behavior of the damping near the interface are similar to what Mills ${ }^{9}$ and Simanek ${ }^{11}$ found and is non-existent in a large sample as is clear from the figure for large $D$. The dimensionless Gilbert damping for iron would be therefore

$$
\alpha=\frac{G_{s}}{\gamma M_{s}} \approx 0.01,
$$

which has the right order of magnitude as measured in ref. 27. Figure 8 is perhaps the case that applies to transition metals. In this case the oscillations are almost nonexistent and is consistent with the recent numerical calculations of Zwierzycki et al. ${ }^{31}$ which are based on the circuit theory approach. ${ }^{32}$ The temperature dependence is weak in all the results since we have assumed that $k_{B} T<<\epsilon_{F}$. For large thicknesses, the damping is therefore still dependent on the exchange coupling and this dependence is linear. This is in contrast to Berger's ${ }^{5}$ result where his interfacial damping is independent of $J$.

Finally, we show that the term $\wedge_{1}$ vanishes to lowest order. To find an explicit expression for the $\Lambda_{1}$-term, we proceed along similar lines as we did for $\Lambda_{2}$. We make a slow time 


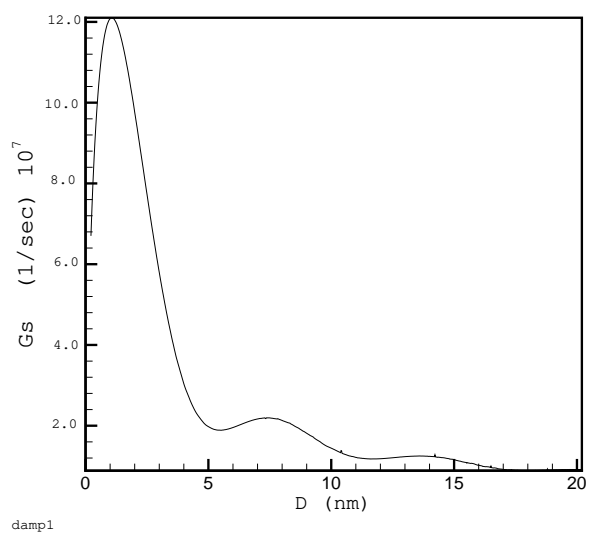

FIG. 5: The damping constant (in $\sec ^{-1}$ ) as a function of the thickness of the ferromagnetic film (in $n m$ ). $k_{F}=10^{8} \mathrm{~cm}^{-1}, \delta_{r}=0.2, \eta_{r}=0.001$

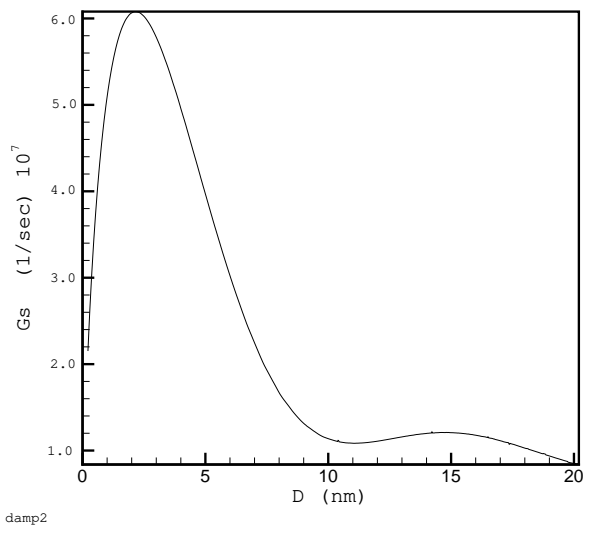

FIG. 6: The damping constant (in $\sec ^{-1}$ ) as a function of the thickness of the ferromagnetic film (in $n m$ ). $k_{F}=10^{8} \mathrm{~cm}^{-1}, \delta_{r}=0.1, \eta_{r}=0.001$.

approximation for the cosine-term in eq. 50 and we define a 'susceptibility' function $\chi^{\prime}(\Omega, \mathbf{p})$ similar to $\chi$,

$$
\begin{aligned}
\chi^{\prime}(\Omega, \mathbf{p})= & -i J^{2} \sum_{\mathbf{k}}\left(f\left(\varepsilon_{1}(\mathbf{k})\right)-f\left(\varepsilon_{2}(\mathbf{k}+\mathbf{p})\right)\right) \\
& \times\left[\frac{1}{\varepsilon_{1}(\mathbf{k})-\varepsilon_{2}(\mathbf{k}+\mathbf{p})+\Omega+i \eta}-\frac{1}{\varepsilon_{1}(\mathbf{k})-\varepsilon_{2}(\mathbf{k}+\mathbf{p})-i \eta}\right] .
\end{aligned}
$$

As in the $\Lambda_{1}$ case, $\Lambda_{2}$ is proportional to the imaginary part of $d \chi^{\prime}(\Omega=0, \mathbf{p}) / d \Omega$ which is easily seen to vanish. At higher orders in $J^{2}$ it gives a nonzero contribution to the frequency. 


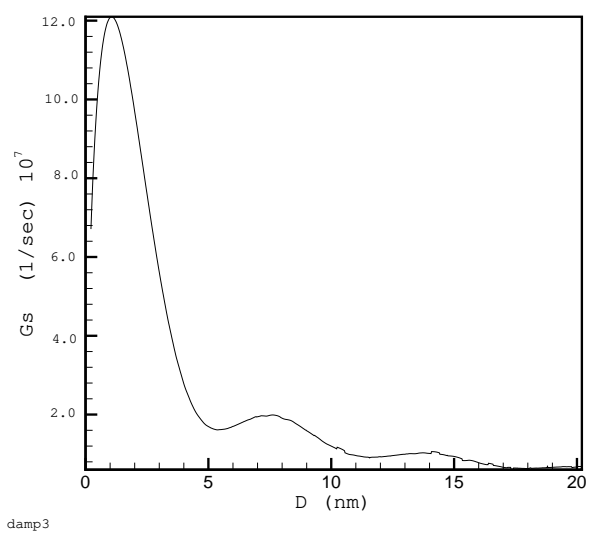

FIG. 7: The damping constant (in $\sec ^{-1}$ ) as a function of the thickness of the ferromagnetic film (in $n m$ ). $k_{F}=10^{8} \mathrm{~cm}^{-1}, \delta_{r}=0.2, \eta_{r}=0.001$

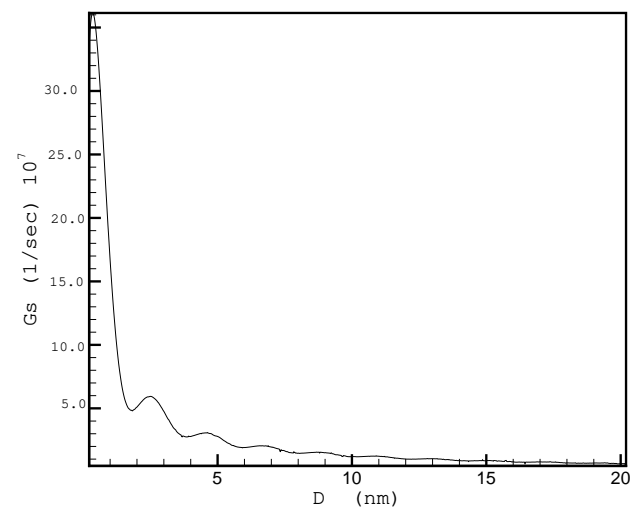

FIG. 8: The damping constant (in $\sec ^{-1}$ ) as a function of the thickness of the ferromagnetic film (in $n m$ ). $k_{F}=10^{8} \mathrm{~cm}^{-1}, \delta_{r}=0.6, \eta_{r}=0.001$

This is the subject of next section.

\section{THE MAGNETIC NOISE SPECTRUM}

In this section, we calculate the various correlation functions of the magnetization vector by including higher order corrections in $J$ in the exchange field. We will deal with both the low frequency limit and the high frequency regime. The former is applicable to the 
case of large magnetization while the latter is important for local atomic moments. We will show that the damping for local moments depends on the symmetry of the Hamiltonian and will not be of the Gilbert-form. In the adiabatic limit which applies to the average magnetization of the film, the GB equation is recovered.

This is a direct extension of the calculations presented in the previous sections. In the bulk it was shown by Heinrich, Fraitova and Kambersky ${ }^{15}$ that the sd-exchange gives zero damping unless dissipation to the phonons is included. This was done in a non-self-consistent way by putting a relaxation time $\tau_{s}$ by hand in the electron propagator. However, based on a simple analogy with Migdal's theory ${ }^{33}$ on electrons and phonons, we should expect a nonzero damping for spin waves with wave numbers $p$ such that $0<k_{F}^{\uparrow}-k_{F}^{\downarrow}<p<k_{F}^{\uparrow}+k_{F}^{\downarrow}$ if $k_{B} T<<\epsilon_{F}$ (fig. 9). Hence the volume mode, i.e, $p=0$ mode, won't be expected to show any dissipation in the bulk. Sine we are partly interested in long-wavelength excitations in thin films, we will concentrate on the consequences of interfacial effects which based on the calculations above should provide a new source for dissipation. We follow closely Schwinger's original work on the harmonic oscillator interacting with a bath of harmonic oscillators. ${ }^{24}$ Some of the computations will be deferred to the appendix in the hope not to distract the reader from the end results. Our treatment is self-contained. Similar calculations have been carried out in Ref. 4 which dealt with a hypothetical physical model for dissipation in the bulk. However in the adiabatic limit, we will recover the results in 4 . This shows that the macroscopic magnetization as opposed to the local moments is insensitive to the dynamics of the environment. In this section, the bulk spin relaxation time $\tau_{s}=0$. We go beyond the mean field approximation for the exchange field. ${ }^{34}$ In other words, we seek corrections to the propagators of the theory by including self-energy corrections. This will allow us to go beyond the approximations made in the previous sections and calculate the damping due to inelastic scattering of the conduction electrons off the magnons as they cross the interfaces. We do not use the slow-time approximation in this section, but the damping will be shown to have the Gilbert form for frequencies much smaller than the electronic precessional frequency. Moreover, we find a very interesting result that relates the GB equation for spin-momentum transfer to the model treated here. We show that the Langevin dynamic treatment of $\mathrm{Li}$ and Zhang ${ }^{35}$ for the noise can be only justified for the adiabatic limit, and around the FMR frequency in thin films. As in the previous section, the magnetization precesses around an effective in-plane field directed along the z-axis. We 


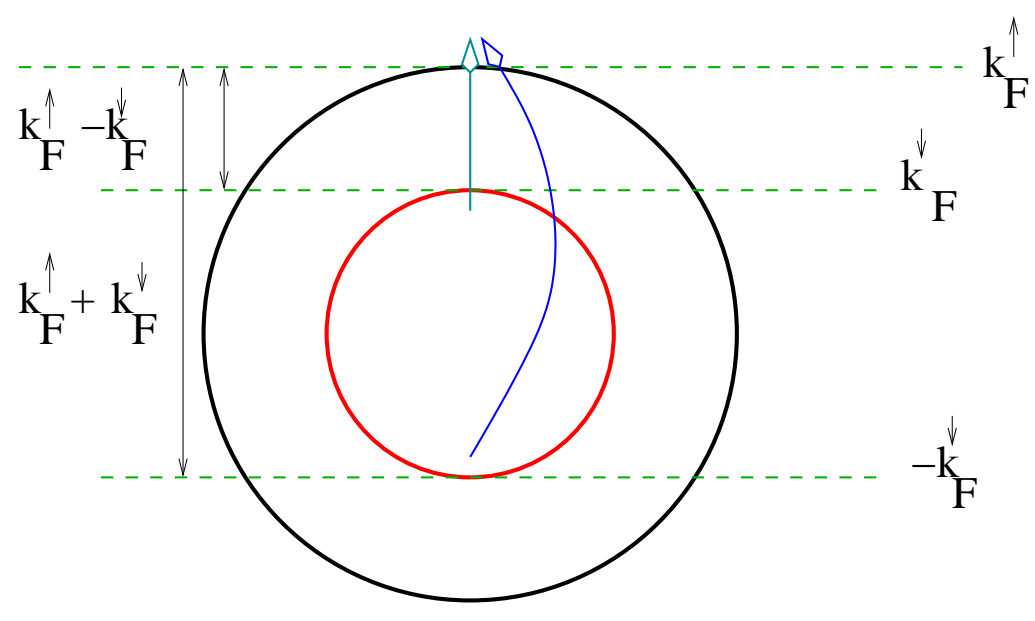

FIG. 9: A spin-down/spin-up excitation in a bulk ferromagnet with smallest and largest wavevectors.

start by coupling the transverse magnetization $\mathbf{S}=\left(S_{x}, S_{y}\right)$ to external sources $\mathbf{J}_{1}$ and $\mathbf{J}_{2}$ along the positive and negative-time oriented paths, respectively. The modified Hamiltonian is now given by

$$
\mathcal{H}_{P}=\mathcal{H}_{1}-\mathbf{J}_{1} \cdot \mathbf{S}_{1}-\left(\mathcal{H}_{2}-\mathbf{J}_{2} \cdot \mathbf{S}_{2}\right) \text {. }
$$

Next we introduce the new variables $\mathbf{J}=\frac{1}{2}\left(\mathbf{J}_{1}+\mathbf{J}_{2}\right)$ and $\mathbf{Q}=\mathbf{J}_{1}-\mathbf{J}_{2}$. Similarly, we define an average $\mathbf{S}=\frac{1}{2}\left(\mathbf{S}_{1}+\mathbf{S}_{2}\right)$ and a difference $\mathbf{D}=\mathbf{S}_{1}-\mathbf{S}_{2}$ representing the fluctuations in the magnetization. The generating functional $\mathbb{Z}\left[\mathbf{J}_{1}, \mathbf{J}_{2}\right]$ is defined in such way that its Taylor expansion at $\mathbf{J}_{1}=\mathbf{J}_{2}=0$ gives the correlation functions of the magnetization,

$$
\mathbb{Z}\left[\mathbf{J}_{1}, \mathbf{J}_{2}\right]=\operatorname{Tr}\left(\rho T^{-1} \exp \left[i \int_{t_{0}}^{t_{f}} d t \mathcal{H}\left[\mathbf{J}_{1}\right]\right] T \exp \left[-i \int_{t_{0}}^{t_{f}} d t \mathcal{H}\left[\mathbf{J}_{2}\right]\right]\right) .
$$

Then we see from the definitions that

$$
\left.\frac{\delta \log \mathbb{Z}\left[\mathbf{J}_{1}, \mathbf{J}_{2}\right]}{\delta \mathbf{J}_{1}(t)}\right|_{J_{1}=J_{2}=0}=i\left\langle\mathbf{S}_{1}(t)\right\rangle,
$$

and

$$
\begin{aligned}
\frac{\delta\left\langle S_{i}(t)\right\rangle}{\delta Q_{j}\left(t^{\prime}\right)} & =i\left\langle S_{i}(t) S_{i}(t)\right\rangle \\
& =\frac{i}{2}\left\langle\left\{\widehat{S}_{i}(t), \widehat{S}_{j}\left(t^{\prime}\right)\right\}\right\rangle .
\end{aligned}
$$

The latter symmetric average is the one usually associated with the noise in the magnetization vector. We will seek a general expression for this quantity that takes into account 
initial conditions, i.e., the reservoirs and the film are assumed initially to be separately in equilibrium before they are put in contact with each other at time $t_{0}$. Hence our method is capable of handling transient behavior in our system. To calculate the symmetric correlation

function, we need first to find the equation of motion of the average value $\langle\mathbf{S}\rangle$. For this, we need the effective action in the presence of the external fields which is easily found from Eq. 47

$$
\begin{aligned}
i S_{\text {eff }}\left[\mathfrak{Z}^{*}, \mathfrak{Z}, \mathfrak{z}^{*}, \mathfrak{z}\right] & =\sum_{\mathbf{k}} \int_{t_{0}}^{t_{f}} d t\left[-\frac{1}{2} \mathfrak{Z}^{*}(\mathbf{k}) \partial_{t} \mathfrak{z}(\mathbf{k})-\frac{1}{2} \mathfrak{z}^{*}(\mathbf{k}) \partial_{t} \mathfrak{Z}(\mathbf{k})\right. \\
& +\frac{1}{2} \mathfrak{Z}(\mathbf{k}) \partial_{t} \mathfrak{z}^{*}(\mathbf{k})+\frac{1}{2} \mathfrak{z}(\mathbf{k}) \partial_{t} \mathfrak{Z}^{*}(\mathbf{k})-i \Omega\left(\mathfrak{Z}^{*}(\mathbf{k}) \mathfrak{z}(\mathbf{k})+\mathfrak{z}^{*}(\mathbf{k}) \mathfrak{Z}(\mathbf{k})\right) \\
& \left.-i K\left(\mathfrak{z}^{*}(\mathbf{k}) \mathfrak{Z}^{*}(-\mathbf{k})+\mathfrak{z}(\mathbf{k}) \mathfrak{Z}(-\mathbf{k})\right)\right] \\
& +J^{\prime 2} \sum_{\mathbf{k}, \mathbf{k}^{\prime}} \int d t d t^{\prime} e^{-i \varepsilon_{1,2}\left(\mathbf{k}, \mathbf{k}^{\prime}\right)\left(t-t^{\prime}\right)}\left[\mathfrak{Z}^{*}(t, \mathbf{p}) \mathcal{U}_{12}\left(t-t^{\prime}\right) \mathfrak{z}\left(t^{\prime},-\mathbf{p}\right)\right. \\
& \left.+\mathfrak{z}^{*}(t, \mathbf{p}) \mathcal{U}_{21}\left(t-t^{\prime}\right) \mathfrak{Z}(t,-\mathbf{p})+\mathfrak{z}^{*}(t, \mathbf{p}) \mathcal{U}_{22}\left(t, t^{\prime}\right) \mathfrak{z}\left(t^{\prime},-\mathbf{p}\right)\right] \\
& +i \sum_{\mathbf{k}} \int d t\left(Q_{c}^{*} \mathfrak{Z}+Q_{c} \mathfrak{Z}^{*}\right)+i \sum_{\mathbf{k}} \int d t\left(J_{c}^{*} \mathfrak{z}+J_{c} \mathfrak{z}^{*}\right)
\end{aligned}
$$

where we have $\mathbf{p}=\mathbf{k}^{\prime}-\mathbf{k}$ and $\varepsilon_{12}\left(\mathbf{k}, \mathbf{k}^{\prime}\right)=\varepsilon_{1}(\mathbf{k})-\varepsilon_{2}\left(\mathbf{k}^{\prime}\right)$. The remaining variables are defined as follows:

$$
\begin{aligned}
\mathfrak{Z} & =\frac{1}{\sqrt{2}}\left(S_{x}+i S_{y}\right), \\
\mathfrak{z} & =\frac{1}{\sqrt{2}}\left(D_{x}+i D_{y}\right), \\
Q_{c} & =\frac{1}{\sqrt{2}}\left(Q_{x}+i Q_{y}\right), \\
J_{c} & =\frac{1}{\sqrt{2}}\left(J_{x}+i J_{y}\right) .
\end{aligned}
$$

Varying the action with respect to its variables, we get the respective equations of motion for the magnetization and the fluctuations. A solution of these equations will require a careful treatment of the boundary conditions. To do this, we found it easier to take a related representation of the coherent states. This representation is equivalent to the usual harmonic oscillator representation.

We define two harmonic oscillator-type operators $a$ and $a^{+}$such that 


$$
\begin{array}{r}
a=\frac{\sqrt{2}}{2}\left[\left(\frac{c_{x x}}{c_{y y}}\right)^{1 / 4} \widehat{S}_{x}+i\left(\frac{c_{y y}}{c_{x x}}\right)^{1 / 4} \widehat{S}_{y}\right], \\
a^{+}=\frac{\sqrt{2}}{2}\left[\left(\frac{c_{x x}}{c_{y y}}\right)^{1 / 4} \widehat{S}_{x}-i\left(\frac{c_{y y}}{c_{x x}}\right)^{1 / 4} \widehat{S}_{y}\right] .
\end{array}
$$

Hence, the energy operator for the spin system becomes

$$
\widehat{H}=\hbar \omega_{0}\left(a^{+} a+\frac{1}{2}\right)
$$

with the frequency given by

$$
\omega_{0}=S \sqrt{c_{x x} c_{y y}} ; \quad(S=1)
$$

Therefore coherent states in this representation are defined by

$$
a|Z\rangle=Z|Z\rangle
$$

To change between representations $(\mathfrak{Z}, \mathfrak{z}) \rightarrow(Z, z)$ ( with careful handling of the boundary conditions) in the path integral representations we need to make the change of variables

$$
\begin{aligned}
\mathfrak{Z} & \rightarrow \frac{1}{2}\left(c_{1} Z+c_{2} Z^{*}\right), \\
\mathfrak{z} & \rightarrow \frac{1}{2}\left(c_{1} z+c_{2} z^{*}\right),
\end{aligned}
$$

with the coefficients $c_{1}$ and $c_{2}$ given by

$$
\begin{aligned}
& c_{1}=\frac{\sqrt{c_{x x}}+\sqrt{c_{y y}}}{2 \sqrt{\omega_{0}}}, \\
& c_{2}=-\frac{\sqrt{c_{x x}}-\sqrt{c_{y y}}}{2 \sqrt{\omega_{0}}} .
\end{aligned}
$$

It will be seen that in the high frequency regime, the damping depends separately on $c_{1}$ and $c_{2}$. The path integral representation on the closed-time path requires doubling of variables as has been done above. The following boundary conditions are also needed:

$$
\begin{aligned}
& Z_{1}\left(t_{f}\right)=Z_{2}\left(t_{f}\right), \\
& Z_{1}\left(t_{0}\right)=\exp \left[-\beta \omega_{0}\right] Z_{2}\left(t_{0}\right), \\
& Z_{1}^{*}\left(t_{0}\right)=\exp \left[+\beta \omega_{0}\right] Z_{2}^{*}\left(t_{0}\right) .
\end{aligned}
$$


It is important to observe that the boundary conditions are non-hermitian in the coherentstate formulation. This is a direct result of the application of the Kubo-Martin-Schwinger (KMS) condition. This implies that $Z$, the average sum of $Z_{1}$ and $Z_{2}$, and their respective fluctuations $z$ satisfy the conditions

$$
Z\left(t_{0}\right)=-\frac{1}{2} \operatorname{coth}\left(\beta \frac{\omega_{0}}{2}\right) z\left(t_{0}\right)
$$

and

$$
z\left(t_{f}\right)=0
$$

The free action in the new representation therefore takes the form

$$
\begin{aligned}
i S^{(0)}\left[Z^{*}, Z, z^{*}, z\right] & =\frac{1}{2}\left(Z_{f} Z_{f}^{*}\left(t_{f}\right)+Z_{f}^{*} Z_{f}\left(t_{f}\right)\right)+\frac{1}{2}\left(Z_{i} Z^{*}\left(t_{0}\right)+Z_{i}^{*} Z\left(t_{0}\right)\right) \\
& +\frac{1}{4}\left(Z_{i} z^{*}\left(t_{0}\right)+Z_{i}^{*} z\left(t_{0}\right)\right) \\
& +i \int_{t_{0}}^{t_{f}} d t\left\{\frac{1}{2 i}\left(Z^{*} z-Z^{*} \dot{z}\right)+\frac{1}{2 i}\left(z^{*} Z-z^{*} \dot{Z}\right)\right. \\
& \left.-\omega_{0}\left(z^{*} Z+z Z^{*}\right)+Z^{*} \Delta+Z \Delta^{*}+z^{*} \Sigma+z \Sigma^{*}\right\}
\end{aligned}
$$

where the external sources $\Delta, \Sigma$ are defined through the relation

$$
\mathbf{J}_{1} \cdot \mathbf{S}_{1}-\mathbf{J}_{2} \cdot \mathbf{S}_{2}=Z^{*} \Delta+Z \Delta^{*}+z^{*} \Sigma+z \Sigma^{*}
$$

The equations of motion are obtained from the full action $i S^{\operatorname{eff}}\left[Z^{*}, Z, z^{*}, z\right]=$ $i S^{(0)}\left[Z^{*}, Z, z^{*}, z\right]+i S^{B}\left[Z^{*}, Z, z^{*}, z\right]$, where the last term is due to the interaction of the conduction electrons with the magnetization. For the fluctuations $z$ and $z^{*}$, we obtain

$$
\begin{gathered}
\left(\partial_{t}+i \omega_{0}\right) z(t, \mathbf{p})-\frac{J^{2}}{4} \int d t^{\prime} G_{1}\left(t-t^{\prime}, \mathbf{p}\right) z\left(t^{\prime},-\mathbf{p}\right) \\
-\frac{J^{2}}{4} \int d t^{\prime} G_{2}\left(t-t^{\prime}, \mathbf{p}\right) z^{*}\left(t^{\prime},-\mathbf{p}\right) \\
=i \Delta(t, \mathbf{p})
\end{gathered}
$$

and

$$
\begin{aligned}
\left(\partial_{t}-i \omega_{0}\right) z^{*}(t, \mathbf{p}) & +\frac{J^{2}}{4} \int d t^{\prime} G_{3}\left(t-t^{\prime}, \mathbf{p}\right) z^{*}\left(t^{\prime},-\mathbf{p}\right) \\
& +\frac{J^{2}}{4} \int d t^{\prime} G_{2}\left(t-t^{\prime}, \mathbf{p}\right) z^{*}\left(t^{\prime},-\mathbf{p}\right) \\
=-i \Delta^{*}(t, \mathbf{p}) . &
\end{aligned}
$$


To solve these equations and take account of the finite size of the film, we introduce the following definition for the 'averaged' Green functions,

$$
\bar{G}_{\alpha}\left(t-t^{\prime}\right)=\frac{1}{D A} \int d^{2} x_{\|} \int d^{2} y_{\|} \int_{-D / 2}^{D / 2} d x e^{i p x} \int_{-D / 2}^{D / 2} d y e^{i p y} \int \frac{d^{3} p}{(2 \pi)^{3}} G_{\alpha}\left(t-t^{\prime}, \mathbf{p}\right) .
$$

where $A$ is the area of the surface of the film. This definition is useful for solving for the zero mode only. As we did in the previous section, this is obtained by Fourier transforming back the equations of motion to the real space representation where the condition of finite thickness can be easily implemented. These Green functions $(\alpha=1, . ., 5)$ are given explicitly in the appendix. It should be noted that the Green functions $\bar{G}_{\alpha}$ with $\alpha=1,2,3$ are directly related to the appearance of the dissipative term in the magnetization. They involve terms similar to those that appeared in Eq. 71, but they are also the Green functions that appear in the equations of motion of the fluctuations $z$ and $z^{*}$. Hence their dissipative nature is very clear in this formalism since they introduce irreversibility in the dynamics of $Z$ and $Z^{*}$.

The corresponding Green's function needed for the solution of the fluctuations are therefore given by

$$
\begin{aligned}
\left(\partial_{t}+i \omega_{0}\right) \mathfrak{g}_{1}\left(t-t^{\prime}\right)-\frac{J^{2}}{4} \int d t^{\prime \prime} \bar{G}_{1}\left(t-t^{\prime \prime}\right) \mathfrak{g}_{1}\left(t^{\prime \prime}-t^{\prime}\right) & =\delta\left(t-t^{\prime}\right), \\
\mathfrak{g}_{1}\left(t-t^{\prime}\right) & =0 ; \quad t>t^{\prime}
\end{aligned}
$$

and

$$
\begin{aligned}
\left(\partial_{t}+i \omega_{0}\right) \mathfrak{g}_{1}^{\prime}\left(t-t^{\prime}\right)+\frac{J^{2}}{4} \int d \tau \bar{G}_{1}(t-\tau) \mathfrak{g}_{1}^{\prime}\left(\tau-t^{\prime}\right) & =\delta\left(t-t^{\prime}\right), \\
\mathfrak{g}_{1}^{\prime}\left(t-t^{\prime}\right) & =0 ; \quad t<t^{\prime}
\end{aligned}
$$

Similarly, we get two more equations for $Z(t)$ and $Z^{*}(t)$ that involve two more Green functions. We only write the respective solutions below. Therefore, the solutions of the fluctuations and the average sum take the form

$$
\begin{aligned}
z(t) & =i \int \mathfrak{g}_{1}\left(t-t^{\prime}\right) \Delta\left(t^{\prime}\right) d t^{\prime} \\
& -i\left(\frac{J}{2}\right)^{2} \int d \tau d t^{\prime} d t^{\prime \prime} \mathfrak{g}_{1}\left(t-t^{\prime}\right) \bar{G}_{2}\left(t-t^{\prime \prime}\right) \mathfrak{g}_{1}^{*}\left(t^{\prime \prime}-\tau\right) \Delta^{*}(\tau),
\end{aligned}
$$


and

$$
\begin{aligned}
Z(t) & =i \int d t^{\prime} \mathfrak{g}_{1}^{\prime}\left(t-t^{\prime}\right) \Sigma\left(t^{\prime}\right)+i \frac{J^{2}}{4} \int d \tau d \tau^{\prime} d t^{\prime} \mathfrak{g}_{1}^{\prime}(t-\tau) \bar{G}_{4}\left(\tau-t^{\prime}\right) \mathfrak{g}_{1}\left(t^{\prime}-\tau\right) \Delta\left(\tau^{\prime}\right) \\
& -i \frac{J^{2}}{4} \int d \tau d \tau^{\prime} d t^{\prime} \mathfrak{g}_{1}^{\prime}(t-\tau) \bar{G}_{5}\left(\tau-t^{\prime}\right) \mathfrak{g}_{1}^{*}\left(t^{\prime}-\tau\right) \Delta^{*}\left(\tau^{\prime}\right) \\
& -i \frac{J^{2}}{4} \int d \tau d \tau^{\prime} d t^{\prime} \mathfrak{g}_{1}^{\prime}(t-\tau) \bar{G}_{2}\left(\tau-t^{\prime}\right) \mathfrak{g}_{1}^{\prime *}\left(t^{\prime}-\tau\right) \Sigma^{*}\left(\tau^{\prime}\right)
\end{aligned}
$$

Since $z\left(t_{f}\right)=0$, we require that $\mathfrak{g}_{1}\left(t-t^{\prime}\right)=0$ for $t>t^{\prime}$.

First, we recalculate the initial correlations to show that we have the correct boundary conditions. The initial value for $Z$ follows from the solution for $Z(t)$ after setting the coupling constant $J=0$

$$
Z\left(t_{0}\right)=-\frac{1}{2} \operatorname{coth}\left(\beta \frac{\omega_{0}}{2}\right) i \int d t^{\prime} \bar{g}_{1}\left(t_{0}-t^{\prime}\right) \Delta\left(t^{\prime}\right)
$$

Then the derivative of the $\mathrm{x}$-component of the magnetization with respect of the external sources is

$$
\frac{1}{i} \frac{\delta \widehat{S}_{x}(t)}{\delta \Delta J_{x}\left(t^{\prime}\right)}=-\frac{1}{2^{2}} \frac{c_{y y}}{\omega_{0}} \operatorname{coth}\left(\beta \frac{\omega_{0}}{2}\right)\left[\mathfrak{g}_{1}\left(t-t^{\prime}\right)+\mathfrak{g}_{1}^{*}\left(t-t^{\prime}\right)\right]
$$

In the free theory, the propagator $\mathfrak{g}_{1}$ is simply given by

$$
\mathfrak{g}_{1}\left(t-t^{\prime}\right)=-\Theta\left(t^{\prime}-t\right) e^{-i \omega_{0}\left(t-t^{\prime}\right)}
$$

Hence

$$
\begin{aligned}
\frac{1}{i} \frac{\delta\left\langle\widehat{S}_{x}\right\rangle}{\delta \Delta J_{x}(t)} & =\frac{1}{2} \frac{c_{y y}}{\omega_{0}} \operatorname{coth}\left(\beta \frac{\omega_{0}}{2}\right) \cos \omega_{0} t \\
& =\frac{1}{2}\left\langle\left\{S_{x}, S_{x}(t)\right\}\right\rangle,
\end{aligned}
$$

which is the desired relation that was derived in Sect. II by a different method. To get this solution, it was crucial that we apply the correct boundary conditions on $Z$ and $z$, Eqs. 96-100.

For the coupled case, the initial condition for the $Z(t)$ equation is

$$
\begin{aligned}
Z\left(t_{0}\right) & =-\frac{1}{2} \operatorname{coth}\left(\beta \frac{\omega_{0}}{2}\right)\left\{-i \int_{t_{0}}^{\infty} d t^{\prime} \mathfrak{g}_{1}\left(t_{0}-t^{\prime}\right) \Delta\left(t^{\prime}\right)\right. \\
& -i\left(\frac{J}{2}\right)^{2} \int_{t_{0}}^{\infty} d \tau d t^{\prime} d t^{\prime \prime} \mathfrak{g}_{1}\left(t-t^{\prime}\right) \bar{G}_{2}\left(t-t^{\prime \prime}\right) \mathfrak{g}_{1}^{*}\left(t^{\prime \prime}-\tau\right) \Delta^{*}(\tau)
\end{aligned}
$$


Hence the general solution for $Z(t)$ is

$$
\begin{aligned}
Z(t) & =Z_{0}(t)+i \int_{t_{0}}^{\infty} d \tau \mathfrak{g}_{1}^{\prime}(t-\tau) \Sigma(\tau) \\
& +i\left(\frac{J}{2}\right)^{2} \int_{t_{0}}^{\infty} d \tau d \tau^{\prime} d t^{\prime} \mathfrak{g}_{1}^{\prime}\left(t-\tau^{\prime}\right) \bar{G}_{4}(t-\tau) \mathfrak{g}_{1}\left(t^{\prime}-\tau^{\prime}\right) \Delta\left(\tau^{\prime}\right) \\
& -i\left(\frac{J}{2}\right)^{2} \int_{t_{0}}^{\infty} d \tau d \tau^{\prime} d t^{\prime} \mathfrak{g}_{1}^{\prime}(t-\tau) \bar{G}_{5}\left(\tau-t^{\prime}\right) \mathfrak{g}_{1}^{*}\left(t^{\prime}-\tau^{\prime}\right) \Delta^{*}\left(\tau^{\prime}\right) \\
& -i\left(\frac{J}{2}\right)^{2} \int_{t_{0}}^{\infty} d \tau d \tau^{\prime} d t^{\prime} \mathfrak{g}_{1}^{\prime}(t-\tau) \bar{G}_{2}\left(\tau-t^{\prime}\right) \mathfrak{g}_{1}^{*^{\prime}}\left(t^{\prime}-\tau^{\prime}\right) \Sigma^{\star}\left(\tau^{\prime}\right)
\end{aligned}
$$

where $Z_{0}(t)$ is a particular solution of the nonhomogeneous problem

$$
\begin{aligned}
Z_{0}(t) & =Z_{0}(t) e^{-i \omega_{0}\left(t-t_{0}\right)}+\left(\frac{J}{2}\right)^{2} e^{-i \omega_{0} t} \int_{t_{0}}^{t} d t^{\prime} e^{i \omega_{0} t^{\prime}}\left[-\int d t^{\prime \prime} \bar{G}_{1}\left(t^{\prime}-t^{\prime \prime}\right) e^{-i \omega_{0}\left(t^{\prime \prime}-t_{0}\right)} Z_{0}\left(t_{0}\right)\right. \\
& \left.\int d t^{\prime \prime} \bar{G}_{2}\left(t^{\prime}-t^{\prime \prime}\right) e^{i \omega_{0}\left(t^{\prime \prime}-t_{0}\right)} \bar{Z}_{0}\left(t_{0}\right)\right]
\end{aligned}
$$

Similarly, we get the solution for $Z^{*}(t)$

$$
\begin{aligned}
Z^{*}(t) & =Z_{0}^{*}\left(t_{0}\right) e^{i \omega_{0}\left(t-t_{0}\right)}-i \int_{t_{0}}^{\infty} d t^{\prime} \mathfrak{g}_{1}^{*^{\prime}}\left(t-t^{\prime}\right) \Sigma^{*}\left(t^{\prime}\right) \\
& -i\left(\frac{J}{2}\right)^{2} \int d \tau d \tau^{\prime} d t^{\prime} \mathfrak{g}_{1}^{*}(t-\tau) \bar{G}_{2}\left(\tau-t^{\prime}\right) \mathfrak{g}_{1}^{\prime}\left(t^{\prime}-\tau^{\prime}\right) \Sigma\left(\tau^{\prime}\right) \\
& -i\left(\frac{J}{2}\right)^{2} \int_{t_{0}} d \tau d \tau^{\prime} d t^{\prime} \mathfrak{g}_{1}^{\prime *}(t-\tau) \bar{G}_{5}\left(\tau-t^{\prime}\right) \mathfrak{g}_{1}\left(t^{\prime}-\tau^{\prime}\right) \Delta\left(\tau^{\prime}\right) \\
& +i\left(\frac{J}{2}\right)^{2} \int_{t_{0}}^{\infty} d \tau d \tau^{\prime} d t^{\prime} \mathfrak{g}_{1}^{\prime}(t-\tau) \bar{G}_{4}\left(\tau-t^{\prime}\right) \mathfrak{g}_{1}^{*}\left(t^{\prime}-\tau^{\prime}\right) \Delta^{*}\left(\tau^{\prime}\right) \\
& +\left(\frac{J}{2}\right)^{2} e^{i \omega_{0}\left(t-t_{0}\right)} \int_{t_{0}}^{t} d t^{\prime} e^{-i \omega_{0}\left(t^{\prime}-t_{0}\right)}\left[\int d t^{\prime \prime} \bar{G}_{3}\left(t-t^{\prime \prime}\right) e^{i \omega_{0}\left(t^{\prime \prime}-t_{0}\right)} Z_{0}^{*}\left(t_{0}\right)\right. \\
& \left.-\int d t^{\prime \prime} \bar{G}_{2}\left(t^{\prime}-t^{\prime \prime}\right) e^{-i \omega_{0}\left(t^{\prime \prime}-t_{0}\right)} Z_{0}\left(t_{0}\right)\right]
\end{aligned}
$$

with the initial state given by

$$
Z_{0}\left(t_{0}\right)=-\frac{1}{2} i \operatorname{coth}\left(\beta \frac{\omega_{0}}{2}\right) \int d t^{\prime} \mathfrak{g}_{1}\left(t_{0}-t^{\prime}\right) \Delta\left(t^{\prime}\right)
$$

Now, it is easy to calculate the components of the magnetization from the above results. We just need to differentiate the average magnetization with respect to $\left(j_{x}, j_{y}\right)$, 


$$
\begin{aligned}
& j_{x}=\frac{\sqrt{2}}{2} \sqrt{\frac{\omega_{0}}{c_{y y}}}\left(\Delta+\Delta^{*}\right), \\
& j_{y}=\frac{\sqrt{2}}{2 i} \sqrt{\frac{\omega_{0}}{c_{x x}}}\left(\Delta-\Delta^{*}\right),
\end{aligned}
$$

to find the symmetric correlation functions for the magnetization.

A straightforward calculation gives the differential of the x-component with respect to $j_{x}$

$$
\begin{aligned}
\frac{1}{i} \frac{\delta\left\langle\widehat{S}_{x}(t)\right\rangle}{\delta j_{x}\left(t^{\prime}\right)} & =\frac{1}{2} \frac{c_{y y}}{\omega_{0}}\left\{-\frac{1}{2} \operatorname{coth}\left(\beta \frac{\omega_{0}}{2}\right)\left[e^{i \omega_{0}\left(t-t_{0}\right)} \mathfrak{g}_{1}^{*}\left(t_{0}-t^{\prime}\right)+e^{-i \omega_{0}\left(t-t_{0}\right)} \mathfrak{g}_{1}\left(t_{0}-t^{\prime}\right)\right]\right. \\
& +\left(\frac{J}{2}\right)^{2} \frac{1}{2} \operatorname{coth}\left(\beta \frac{\omega_{0}}{2}\right)\left[e^{-i \omega_{0}\left(t-t_{0}\right)} \int_{t_{0}}^{t} d \tau \int d \tau^{\prime} e^{i \omega_{0}\left(\tau-t_{0}\right)}\right. \\
& \times\left[\bar{G}_{1}\left(\tau-\tau^{\prime}\right) e^{-i \omega_{0}\left(\tau^{\prime}-t_{0}\right)} \mathfrak{g}_{1}\left(t_{0}-t^{\prime}\right)-\bar{G}_{2}\left(\tau-\tau^{\prime}\right) e^{i \omega_{0}\left(\tau^{\prime}-t_{0}\right)} \mathfrak{g}_{1}^{*}\left(t_{0}-t^{\prime}\right)\right] \\
& -e^{i \omega_{0}\left(t-t_{0}\right)} \int_{t_{0}}^{t} d \tau \int d \tau^{\prime} e^{-i \omega_{0}\left(\tau-t_{0}\right)}\left[\bar{G}_{3}\left(\tau-\tau^{\prime}\right) e^{i \omega_{0}\left(\tau^{\prime}-t_{0}\right)} \mathfrak{g}_{1}^{*}\left(t_{0}-t^{\prime}\right)\right. \\
& \left.\left.-\bar{G}_{2}\left(\tau-\tau^{\prime}\right) e^{-i \omega_{0}\left(\tau^{\prime}-t_{0}\right)} \mathfrak{g}_{1}\left(t_{0}-t^{\prime}\right)\right]\right] \\
& +\left(\frac{J}{2}\right)^{2}\left[\int d \tau d \tau ^ { \prime } \left[\mathfrak{g}_{1}^{\prime}(t-\tau) \bar{G}_{4}\left(\tau-\tau^{\prime}\right) \mathfrak{g}_{1}\left(\tau^{\prime}-t^{\prime}\right)\right.\right. \\
& -\mathfrak{g}_{1}^{*^{\prime}}(t-\tau) \bar{G}_{5}\left(\tau-\tau^{\prime}\right) \mathfrak{g}_{1}\left(\tau^{\prime}-t^{\prime}\right)-\mathfrak{g}_{1}^{\prime}(t-\tau) \bar{G}_{5}\left(\tau-\tau^{\prime}\right) \mathfrak{g}_{1}^{*}\left(\tau^{\prime}-t^{\prime}\right) \\
& \left.\left.\left.+\mathfrak{g}_{1}^{*^{\prime}}(t-\tau) \bar{G}_{4}\left(\tau-\tau^{\prime}\right) \mathfrak{g}_{1}^{*}\left(\tau^{\prime}-t^{\prime}\right)\right]\right]\right\} \\
& =\frac{1}{2}\left\langle\left\{\widehat{S}_{x}(t), \widehat{S}_{x}\left(t^{\prime}\right)\right\}\right\rangle .
\end{aligned}
$$

This is a general result for the noise due to spin-flip scattering between magnons and conduction electrons that is useful for all frequencies. It is clear from this expression, that the correlation functions depend explicitly on the initial state of the system. However, there is a term which is invariant under time translation. This part of the correlation term survives at times much later than the initial conditions. Before we turn to the calculation of this term which is the term usually measured in FMR-type experiments we give the 
expression for the correlation function of $S_{x}$ and $S_{y}$ components of the magnetization

$$
\begin{aligned}
\frac{1}{i} \frac{\delta\left\langle\widehat{S}_{x}(t)\right\rangle}{\delta j_{y}\left(t^{\prime}\right)} & =\frac{i}{2}\left\{-\frac{1}{2} \operatorname{coth}\left(\beta \frac{\omega_{0}}{2}\right)\left[e^{-i \omega_{0}\left(t-t_{0}\right)} \mathfrak{g}_{1}\left(t_{0}-t^{\prime}\right)-e^{i \omega_{0}\left(t-t_{0}\right)} \mathfrak{g}_{1}^{*}\left(t_{0}-t^{\prime}\right)\right]\right. \\
& +\left(\frac{J}{2}\right)^{2}\left[\int d \tau d \tau ^ { \prime } \left[\mathfrak{g}_{1}^{\prime}(t-\tau) \bar{G}_{4}\left(\tau-\tau^{\prime}\right) \mathfrak{g}_{1}\left(\tau^{\prime}-t^{\prime}\right)\right.\right. \\
& -\mathfrak{g}_{1}^{*^{\prime}}(t-\tau) \bar{G}_{5}\left(\tau-\tau^{\prime}\right) \mathfrak{g}_{1}\left(\tau^{\prime}-t^{\prime}\right)+\mathfrak{g}_{1}^{\prime}(t-\tau) \bar{G}_{5}\left(\tau-\tau^{\prime}\right) \mathfrak{g}_{1}^{*}\left(\tau^{\prime}-t^{\prime}\right) \\
& \left.-\mathfrak{g}_{1}^{*^{\prime}}(t-\tau) \bar{G}_{4}\left(\tau-\tau^{\prime}\right) \mathfrak{g}_{1}^{*}\left(\tau^{\prime}-t^{\prime}\right)\right] \\
& +\frac{i}{2}\left(\frac{J}{2}\right)^{2} e^{-i \omega_{0}\left(t-t_{0}\right)} \operatorname{coth}\left(\beta \frac{\omega_{0}}{2}\right) \int_{t_{0}}^{t} d \tau e^{i \omega_{0}\left(\tau-t_{0}\right)} \\
& \times \int d t^{\prime \prime}\left[\bar{G}_{1}\left(\tau-t^{\prime \prime}\right) e^{-i \omega_{0}\left(t^{\prime \prime}-t_{0}\right)} \mathfrak{g}_{1}\left(t_{0}-t^{\prime}\right)+\bar{G}_{2}\left(\tau-t^{\prime \prime}\right) e^{i \omega_{0}\left(t^{\prime \prime}-t_{0}\right)} \mathfrak{g}_{1}^{*}\left(t_{0}-t^{\prime}\right)\right] \\
& +\frac{i}{2}\left(\frac{J}{2}\right)^{2} e^{i \omega_{0}\left(t-t_{0}\right)} \operatorname{coth}\left(\beta \frac{\omega_{0}}{2}\right) \int_{t_{0}}^{t} d \tau e^{-i \omega_{0}\left(\tau-t_{0}\right)} \\
& \left.\times \int d t^{\prime \prime}\left[\bar{G}_{3}\left(\tau-t^{\prime \prime}\right) e^{i \omega_{0}\left(t^{\prime \prime}-t_{0}\right)} \mathfrak{g}_{1}^{*}\left(t_{0}-t^{\prime}\right)+\int d t^{\prime \prime} \bar{G}_{2}\left(\tau-t^{\prime \prime}\right) e^{-i \omega_{0}\left(t^{\prime \prime}-t_{0}\right)} \mathfrak{g}_{1}\left(t_{0}-t^{\prime}\right)\right]\right\}
\end{aligned}
$$

An explicit expression for these correlation functions is not needed for what follows, but we will write its limit in the adiabatic limit which is the case of most current interest for the average magnetization of the film. Before we do that, we would like to make few more comments about these general expressions for the correlation functions. These expressions are beyond the usual fluctuation-dissipation relation and hence they can be adapted to truly 'non-equilibrium' situations. As an example we mention time-dependent pulse-field excitations of the magnetization, switching by a magnetic field. In this latter case, the z-axis is the local equilibrium axis. They also give us an idea on the noise behavior in local magnetic moments and the corresponding damping.

To calculate the noise spectrum in the x-component of the magnetization we need the Fourier component of the function

$$
C_{x x}\left(t-t^{\prime}\right)=\left.\left\langle S_{x}(t) S_{x}\left(t^{\prime}\right)\right\rangle\right|_{t>>t_{0}, t^{\prime}>>t_{0}}
$$

since here we will not address the transient regime, which is treated elsewhere ${ }^{20}$. Its Fourier transform is easily found to be

$$
C_{x x}(\omega)=\frac{c_{y y}}{\omega_{0}}\left(\frac{J}{2}\right)^{2} \operatorname{Re}\left\{\mathfrak{g}_{1}^{\prime}(\omega) \bar{G}_{4}(\omega) \mathfrak{g}_{1}(\omega)-\mathfrak{g}_{1}^{\prime}(\omega) \bar{G}_{5}(\omega) \mathfrak{g}_{1}^{*}(-\omega)\right\}
$$


The damping for large thicknesses $D$ (and nonzero exchange splitting) acquires a simple asymptotic expression,

$$
\alpha \approx \frac{\pi\left(c_{1}^{2}+c_{2}^{2}\right)}{2^{5}\left(k_{F}^{\uparrow}+k_{F}^{\downarrow}\right) D}\left(1+2 \frac{\sin \left(\frac{1}{4}\left(k_{F}^{\uparrow}-k_{F}^{\downarrow}\right) D\right)}{\left(k_{F}^{\uparrow}-k_{F}^{\downarrow}\right) D}+48 \frac{\cos \left(\frac{1}{4}\left(k_{F}^{\uparrow}-k_{F}^{\downarrow}\right) D\right)}{\left(k_{F}^{\uparrow}-k_{F}^{\downarrow}\right)^{2} D^{2}}+\ldots\right)
$$

This damping differs from the one found in the previous sections in two different aspects. The first is that for large thicknesses, the damping is weakly dependent on the sd-exchange energy $\delta_{r}$ as opposed to being linear in $\delta_{r}$. This result is now much similar to Mills and Berger. The second important difference is that the relaxation time depends explicitly on the symmetry of the original Hamiltonian of the magnetization. This result is however similar to what we found in ref. 4. For circular precession, we simply have $c_{1}^{2}+c_{2}^{2}=1$ and hence any dependence on the form of the precession is lost. The damping is still however a scalar of the Gilbert form for $\omega<<\delta / \hbar$ and does not appear to require a tensor form as suggested in ref. 18. The high frequency regime, which is applicable to atomic moments, is however more interesting in this respect. The relaxation time is not a simple function of the ellipticity and hence the damping is not of the Gilbert form; the relaxation time is an algebraic function of the frequency (see appendix). The damping in the film still shows oscillations as a function of the thickness of the film and it attains larger values than in the previous calculations to first order in $J^{2}$ (fig. 10-11).

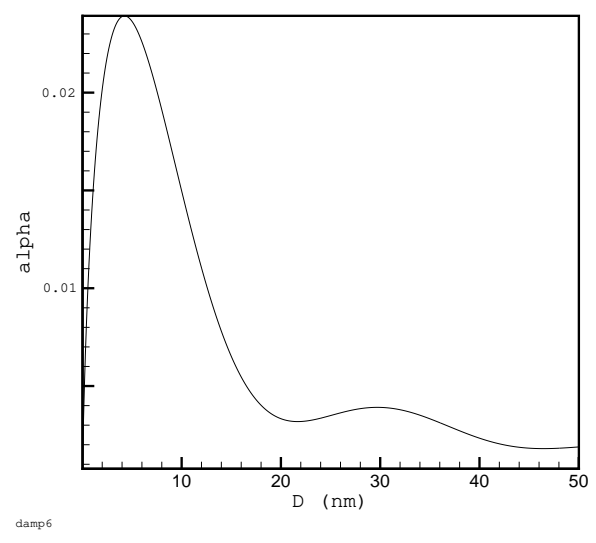

FIG. 10: The damping as a function of the thickness for $\delta_{r}=0.2$ and $c_{x x}=100 c_{y y}$. The last term is a measure of anisotropy and is on the high side for realistic permalloy films. 


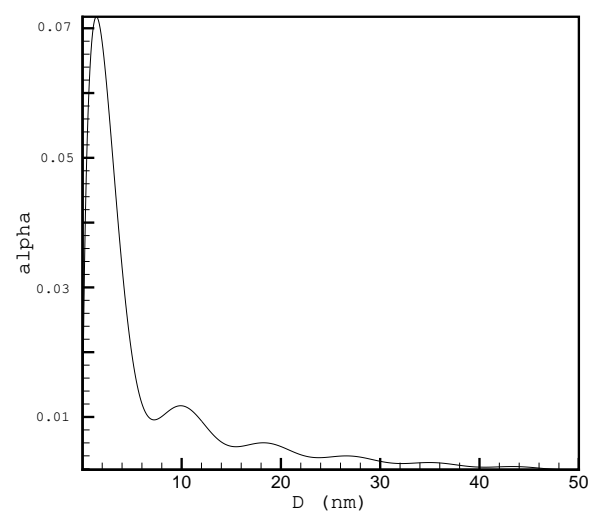

FIG. 11: The damping as a function of the thickness for $\delta_{r}=0.6$ and $c_{x x}=100 c_{y y}$ (same as fig. $10)$.

The expression for the noise, Eq. 127, shows a very interesting property that only the Green functions $\bar{G}_{4}$ and $\bar{G}_{5}$ show up as multiples of the coupling constant $J^{2}$ in the numerator. The dissipative Green functions do not appear in this form. This observation is important when we try to include the effect of spin accumulation on the noise and understand why the effective temperature concept appears in the stochastic formulation of $\mathrm{Li}$ and Zhang ${ }^{35}$ for close to equilibrium. It is also important to observe that in the half-metallic limit, i.e., $J \rightarrow \infty$, we have

$$
\alpha=\frac{\pi\left(c_{1}^{2}+c_{2}^{2}\right)}{2^{6}\left(k_{F} D\right)},
$$

Hence, the exchange energy splitting drops out completely from the damping expression. This result is similar to that derived by Bazaliy, Jones and Zhang ${ }^{36}$ in a half-infinite ferromagnet in contact with a normal metal. In the adiabatic limit, $\omega<<J$, the expression for the noise can be simplified considerably. At high temperature (i.e., $k_{B} T>>w_{0}$ and $T<<T_{c}$ the critical temperature), it becomes

$$
C_{x x}(\omega)=\frac{2 \alpha k_{B} T}{\left(c_{1}^{2}+c_{2}^{2}\right)}\left(\frac{\omega^{2}+c_{y y}+(\alpha \omega)^{2}}{\left[\left(\omega-\omega_{0}\right)^{2}+(\alpha \omega)^{2}\right]\left[\left(\omega+\omega_{0}\right)^{2}+(\alpha \omega)^{2}\right]}\right),
$$

where the damping $\alpha$ is thickness dependent and vanishes when $D \rightarrow \infty$ in the film. The shift in frequency is less than one percent and is neglected in the macroscopic case. A similar result derived in the isotropic case has been recently communicated to us by 37 . A comparison of this expression with the corresponding expression in ref. 3 reveals a very 
interesting result; the dynamic of the d-electrons in the presence of the sd-exchange interaction is simply reproducible by the GB equation with white noise and $\alpha$ is replaced by $\alpha_{G}\left(c_{1}^{2}+c_{2}^{2}\right)$ where $\alpha_{G}$ is the Gilbert damping. This is an important simple result which shows the reasons behind the successes of the GB equation. ${ }^{3}$ At the same time this latter result points to the limits of applicability of the GB equation in atomic simulations. ${ }^{14}$ The recent work of Safonov and Bertram ${ }^{38}$ suggests changing the damping form of the GB equation. Our calculation clearly shows that for the average classical magnetization this is not needed. It is only for high frequencies that the effect of the symmetry of the Hamiltonian on the damping becomes appreciable (see appendix). For low frequencies, the dependence of the relaxation time on the ellipticity factor, $c_{1}^{2}+c_{2}^{2}$, agrees with Kambersky and Patton ${ }^{39}$. In this work, we are also able to give explicit expressions for the damping term and the corresponding noise in thin films. In the next section, we will use the GB equation with a damping $\alpha=0.03$ to study the continuum case. We will also include polarization due to another magnetic layer with relative angle far from zero or $180^{\circ}$. Next we treat the effect of spin accumulation on the noise in the low frequency regime and close to the FMR frequency. As we stated after Eq. 71, the spin accumulation term will have to be included in the self-energy term. The only Green functions where this has to be taken into account are those that appear as a result of the fluctuations $z$ and $z^{*}$. These Green functions will affect only terms where the parameter $\alpha$ and the frequency $\omega$ appear in the Gilbert form in the $\bar{G}_{1}$ Green function only. The overall $\alpha$-term that shows up in front of the temperature will therefore remain unaffected by the spin accumulation term. To show this result, requires a more careful evaluation of the $\bar{G}_{1}(\omega)$. The $\mathbf{k}$-integrals over $\mathbf{k}$ and $\mathbf{k}-\mathbf{p}$ are carried out separately. In the limit of small damping and close to the FMR frequency, we can define an effective temperature in the GB equation

$$
T^{*}=T \frac{1}{\left(1+\frac{\Delta \mu^{\uparrow \downarrow}}{\omega_{0}}\right)},
$$

and a renormalized interfacial damping

$$
\alpha^{*}=\alpha\left(1+\frac{\Delta \mu^{\uparrow \downarrow}}{\omega_{0}}\right) \text {. }
$$

This will allow us to write the corresponding noise in a form that is remarkably similar to that derived recently by $\mathrm{Li}$ and Zhang ${ }^{35}$ where they considered only bulk damping in their problem. However there is a very important difference. The difference in chemical 
potential is expected to be proportional to the current ${ }^{5,7}$ but not linear to the damping as in 35 which is based on the Slonczweski picture. Therefore this expression should be able to differentiate between the sd mechanism and the Slonczewski mechanism. There are already indirect indication from ref. 40 that the critical current is not linear with damping which is the result derived in 35 and includes only bulk damping. In CPP structures therefore we

should not expect the spin momentum transfer term to change the character of noise in these systems as long as we stay below critical currents. There are however other effects in this geometry not addressed by this calculation. One of them is the field from the current and the other is magnon-phonon interactions which my may affect the average magnetization configuration in these films. We discuss these two effects classically in the following section.

\section{DISCUSSION AND CONCLUSION}

In this final section, we would like to make some comments and discuss our results in light of recent experiments on noise in spin valves, such as that in ref. 19, which show $1 / \mathrm{f}$ type noise in CPP structure. In CPP spin valves with biased fields, it was observed that excessive low frequency noise is generated with current. It was argued that spin momentum transfer between the magnetic layers is responsible for this noise. In this section, we treat a case where the spin momentum transfer is not the root cause of noise in these geometries but it will just affect the amplitudes of the noise. The noise in our case will be inherently due to at least three contributing factors: the biasing of the spin valve, the field from the current and the thermal fluctuations in the system. We reach these conclusions based on our calculations carried out in previous sections and on simulations based on the GB equation with a spin torque as it was suggested in ref. 12 ,

$$
\frac{d \mathbf{S}}{d t}=\gamma \mathbf{S} \times\left(\mathbf{H}_{e f f}+\alpha_{G} \frac{\mathbf{d} \mathbf{S}}{d t}+\mathbf{h}\right)+\beta I \mathbf{S} \times\left(\mathbf{S} \times \mathbf{S}_{p}\right)
$$

with the stochastic field $\mathbf{h}(t)$ satisfying

$$
\left\langle h_{i}(t) h_{j}\left(t^{\prime}\right)\right\rangle=2 \alpha_{G} k_{B} T \delta_{i j} \delta\left(t-t^{\prime}\right) .
$$

The parameter $\beta$ is a geometrical factor and $I$ is the current. The pinned layer with magnetization $\mathbf{S}_{p}$ is not dynamical and hence the finite size effects discussed above can 
be included by choosing a large $\alpha_{G}$. The effect of spin accumulation is not taken into account properly in the stochastic field; we are simply assuming the effective $\alpha_{G}$ to be a constant and current independent. This equation is solved numerically with $\alpha_{G}=0.02$, $M_{s}=1400 \mathrm{emu} / \mathrm{cc}$ and thickness $d=3 \mathrm{~nm} \cdot{ }^{41,42}$ The white noise approximation as discussed above is valid for frequencies around the FMR frequency which is of the order of $10 \mathrm{GHz}$. There are two theories of spin momentum transfer: one is microscopic and based on the sdexchange model while the second is macroscopic and is based on a simple balance equation for the spin currents. The calculations presented in previous sections are closer to the first approach rather than to the second one. It is believed that the first approach is dominant only at very thin films of $10 \mathrm{~nm}$ or less. ${ }^{43}$ Therefore based on the results derived here, it appears that any $1 / \mathrm{f}$-type noise measured in $\mathrm{CPP}$ spin valves should be attributed to non sd-type of interactions. We show below that the spin torque does not appear to generate $1 / \mathrm{f}$-type noise and it is the non-homogeneities in the magnetic configurations that are mainly responsible for the noise. The CPP structure we study is shown in fig. 12 and is similar to that in 19. The current is flowing from the pinned to the free layer which are separated by a normal conducting layer. The single particle simulations of the magnetization show no interesting behavior and are noiseless and this is consistent with the results from the previous section. The field from the current is taken into account in the calculations and is needed to observe $1 /$ f-type noise at finite temperature. The magnetization is also biased in the y-axis and $\mathrm{x}$-axis with a 300 Oe field and a -90 Oe field, respectively. The demagnetization field of the sample is also taken into account. The magnetic material is chosen to be that of a permalloy. Figures 13 and 14 clearly show that the effect of the spin torque only slightly increases the already present noise in the system for the particular parameters shown in the figure. It does not give rise to the low frequency in this example. A closer study of this example shows that it is the combination of the biasing, the field from the current and the temperature that are the source of the noise. Hence in this example, neither the sd-type model nor the macroscopic model can explain the origin of the $1 / \mathrm{f}$-type noise. A calculation that does not include in great detail the configuration of the magnetization is therefore highly unlikely to capture the source of the noise in these structures. Figures 16 and 17 show the two possible metastable states that are responsible for the $1 / \mathrm{f}$-type noise in this device. 


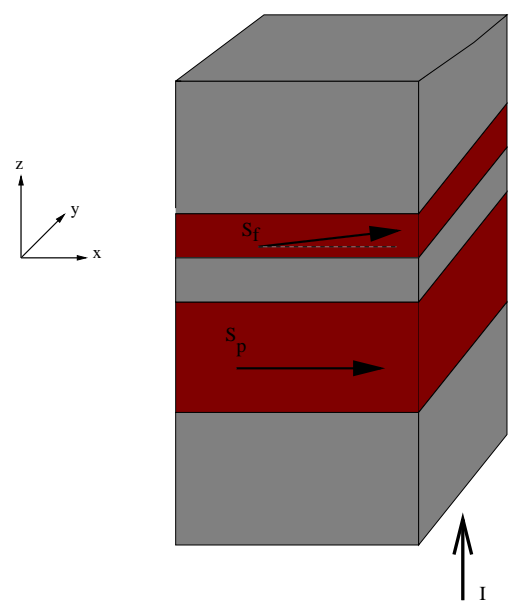

FIG. 12: The CPP spin valve: The thick layer with magnetization $S_{p}$ is pinned along the x-axis. The magnetization $S_{f}$ is free to move. The current I is perpendicular to the interfaces. Spin momentum is transferred from the pinned layer to the layer by polarizing the current with the fixed layer.

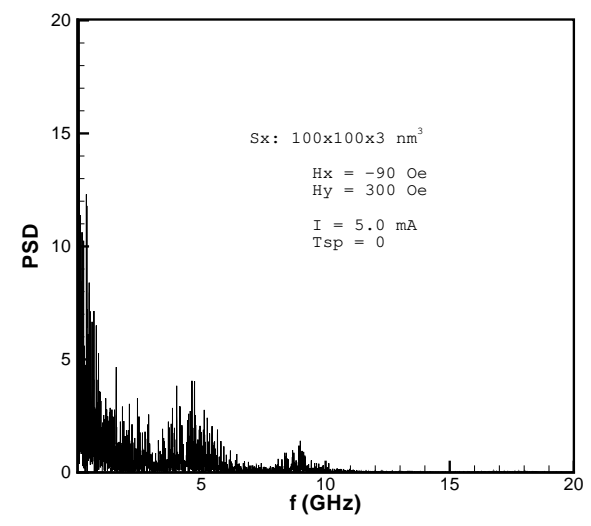

FIG. 13: The xx-component of the noise spectrum for the average $x$-component of the magnetization with the spin torque $T_{s p}$ between the magnetic layers set to zero in the GB equation.

In summary, we have studied in some detail the noise and the damping problem in thin magnetic films embedded between two normal conductors. We have mainly focused on the interaction between the conduction electrons and the d-electrons as the main mechanism for damping. Our results also apply to the microscopic case at high frequencies. In this model, it has been shown that the damping in thin layers oscillates as a function of the 


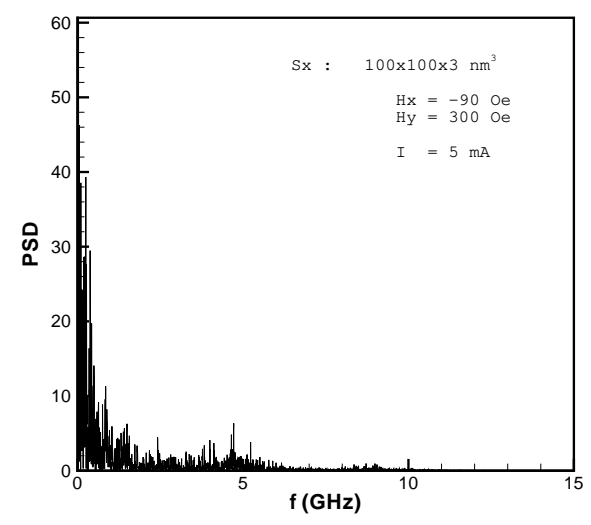

FIG. 14: Same as in Fig. 13 but with the spin momentum transfer torque included in the GB equation.

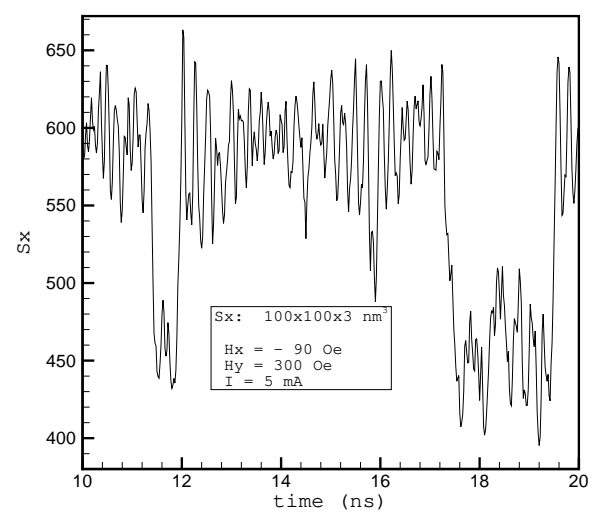

FIG. 15: A real-time trace of the average magnetization component $S_{x}$ (only a typical interval of time is shown). The magnetization appears to oscillate largely in this component for this magnetic configuration where the total magnetization is mainly along the $y$-axis that is perpendicular to the polarization axis.

thickness of the film. If higher orders in the exchange coupling constant are taken into account, the relaxation time becomes dependent on the ellipticity of the precession of the magnetization. Only at high frequencies, the damping is no longer of the Gilbert form and becomes explicitly dependent on the ellipticity. This result is important for atomic simulations. The noise associated with the interfacial damping has also been calculated. It 


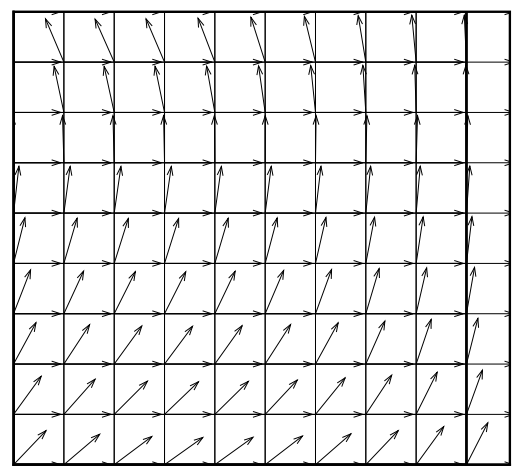

FIG. 16: Configuration of the magnetization in state 1. The horizontal arrows are along $\mathbf{S}_{p}$. The angle between the external bias field and $\mathbf{S}_{p}$ is close to 90 degrees.

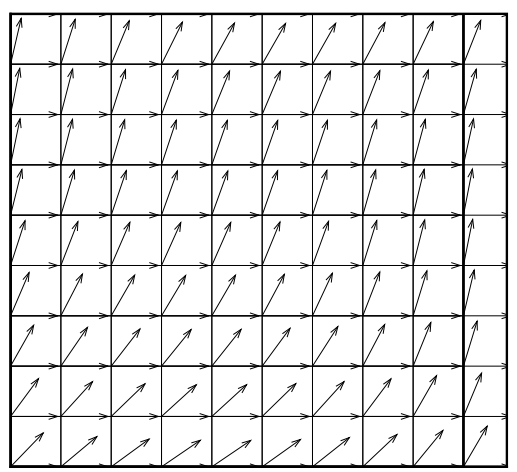

FIG. 17: Configuration of the magnetization in state 2 (same as in fig. 16).

was shown that it does not give rise to a large $1 /$ f-type noise. The spectral density curve gives the usual peak at the natural frequency of the system at low damping. In the adiabatic limit, we have found that for small damping and close to equilibrium, the spectrum is well represented by a white noise source term and an effective temperature that measures deviations from small spin accumulations. Therefore a large negative current can give rise to a large renormalized damping and suppression of the noise amplitude around the FMR peak while positive current decreases the effective damping and increases the noise around the FMR peak. Finally, we have also shown that even within a macroscopic calculation the 
transfer of spin momentum does not give rise to large low frequency noise, rather in our example it was the field from the current, the thermal fluctuations and a particular biasing that simultaneously give rise to large noise at frequencies below the FMR frequency.

\section{acknowledgment}

The first author expresses his warmest thanks to G. Bauer, L. Berger, D. Boyanovsky, M. Covington, S. Mukherjee, C. Patton, E. Rossi, E. Simanek, M. Stiles, V. Safonov, Y. Tserkovnyak and S. Zhang for useful discussions that helped shape the text in the present form. We are also very grateful to D. L. Mills for sending us details about his method of solution used in his paper. (AR) is also very grateful to Dr. R. Chantrell for his encouragement and support to this work. P. Asselin has kindly commented on parts of the manuscript.

\section{APPENDIX A: APPENDIX}

Here we give the definitions of the different functions that appear in the main text and briefly discuss the high frequency regime of the damping term which becomes dependent on the symmetry of the Hamiltonian in a non-trivial way. The following function are derived by integrating out the conduction electrons degrees of freedom from the magnetizationconduction electron equations of motion. The functions are

$$
\begin{aligned}
G_{1}\left(t-t^{\prime}, \mathbf{p}\right) & =\sum_{\mathbf{k}}\left\{c_{1}^{2} \mathcal{U}_{12}\left(t-t^{\prime}\right) e^{-i \varepsilon_{12}(\mathbf{k}+\mathbf{p})\left(t-t^{\prime}\right)}\right. \\
& \left.+c_{2}^{2} \mathcal{U}_{21}\left(t^{\prime}-t\right) e^{i \varepsilon_{12}(\mathbf{k}-\mathbf{p})\left(t-t^{\prime}\right)}\right\} \\
G_{2}\left(t-t^{\prime}, \mathbf{p}\right) & =\sum_{\mathbf{k}}\left\{c_{1} c_{2} \mathcal{U}_{12}\left(t-t^{\prime}\right) e^{-i \varepsilon_{12}(\mathbf{k}+\mathbf{p})\left(t-t^{\prime}\right)}\right. \\
& \left.+c_{1} c_{2} \mathcal{U}_{21}\left(t^{\prime}-t\right) e^{i \varepsilon_{12}(\mathbf{k}-\mathbf{p})\left(t-t^{\prime}\right)}\right\}
\end{aligned}
$$

and

$$
\begin{aligned}
G_{3}\left(t-t^{\prime}, \mathbf{p}\right) & =\sum_{\mathbf{k}}\left\{c_{2}^{2} \mathcal{U}_{12}\left(t-t^{\prime}\right) e^{-i \varepsilon_{12}(\mathbf{k}+\mathbf{p})\left(t-t^{\prime}\right)}\right. \\
& \left.+c_{1}^{2} \mathcal{U}_{21}\left(t^{\prime}-t\right) e^{i \varepsilon_{12}(\mathbf{k}-\mathbf{p})\left(t-t^{\prime}\right)}\right\}
\end{aligned}
$$


are due to the correlation terms between the two branches of the CTP path of the path integral. The following functions are symmetric in time and will be the origin of the correlation functions of the random field:

$$
\begin{aligned}
G_{4}\left(t-t^{\prime}, \mathbf{p}\right) & =\sum_{\mathbf{k}}\left\{c_{1}^{2} \mathcal{U}_{22}(\mathbf{k}+\mathbf{p}) e^{-i \varepsilon_{12}(\mathbf{k}+\mathbf{p})\left(t-t^{\prime}\right)}\right. \\
& \left.+c_{2}^{2} \mathcal{U}_{22}(\mathbf{k}-\mathbf{p}) e^{i \varepsilon_{12}(\mathbf{k}-\mathbf{p})\left(t-t^{\prime}\right)}\right\}, \\
G_{5}\left(t-t^{\prime}, \mathbf{p}\right) & =\sum_{\mathbf{k}}\left\{c_{1} c_{2} \mathcal{U}_{22}(\mathbf{k}+\mathbf{p}) e^{-i \varepsilon_{12}(\mathbf{k}+\mathbf{p})\left(t-t^{\prime}\right)}\right. \\
& \left.+c_{1} c_{2} \mathcal{U}_{22}(\mathbf{k}-\mathbf{p}) e^{i \varepsilon_{12}(\mathbf{k}-\mathbf{p})\left(t-t^{\prime}\right)}\right\}
\end{aligned}
$$

All these functions are not independent. For example, we have

$$
G_{3}\left(t-t^{\prime},-\mathbf{p}\right)=-G_{1}\left(t-t^{\prime}, \mathbf{p}\right)
$$

In most of our calculations, the functions $\mathcal{U}_{22}$ are approximated by the following expressions:

$$
\mathcal{U}_{22}(\mathbf{k}+\mathbf{p})=\frac{1}{2} \delta\left(\varepsilon(\mathbf{k})-\bar{\varepsilon}_{F}\right)\left(\frac{K \cdot \mathbf{p}}{m}+\Delta\right) \operatorname{coth}\left(\frac{\beta}{2}\right)\left(\frac{K \cdot \mathbf{p}}{m}+\Delta\right)
$$

and

$$
\mathcal{U}_{22}(\mathbf{k}-\mathbf{p})=\frac{1}{2} \delta\left(\varepsilon(\mathbf{k})-\bar{\varepsilon}_{F}\right)\left(-\frac{K \cdot \mathbf{p}}{m}+\Delta\right) \operatorname{coth}\left(\frac{\beta}{2}\right)\left(-\frac{K \cdot \mathbf{p}}{m}+\Delta\right)
$$

These approximations are not necessary but makes the algebra less involved.

The function $G_{1}$ has the exact explicit expression:

$$
\begin{aligned}
G_{1}\left(t-t^{\prime}, \mathbf{p}\right) & =\sum_{\mathbf{k}}\left\{-c_{1}^{2} \Theta\left(t-t^{\prime}\right)\left[f_{1}(\mathbf{k})-f_{2}(\mathbf{k}+\mathbf{p})\right] e^{-i \varepsilon_{12}(\mathbf{k}+\mathbf{p})\left(t-t^{\prime}\right)}\right. \\
& \left.+c_{2}^{2} \Theta\left(t^{\prime}-t\right)\left[f_{1}(\mathbf{k})-f_{2}(\mathbf{k}+\mathbf{p})\right] e^{i \varepsilon_{12}(\mathbf{k}-\mathbf{p})\left(t-t^{\prime}\right)}\right\}
\end{aligned}
$$

Similar expressions for $G_{2}$ and $G_{3}$ can be easily deduced from that of $G_{1}$.

All these functions are needed for the calculation of the propagators for the fields $Z, Z^{*}, z$ and $z^{*}$, which are to first order in $J^{2}$ given, respectively by 


$$
\begin{aligned}
\mathfrak{g}_{1}\left(t-t^{\prime}\right) & =\Theta\left(t-t^{\prime}\right) e^{i \omega_{0}\left(t-t^{\prime}\right)} \\
& +\left(\frac{J}{2}\right)^{2} \int d t^{\prime \prime} \int d \tau \Theta\left(t^{\prime \prime}-t\right) e^{i \omega_{0}\left(t-t^{\prime \prime}\right)} \\
& \times \bar{G}_{1}\left(t^{\prime \prime}-\tau\right) \Theta\left(t^{\prime}-\tau\right) e^{i \omega_{0}\left(\tau-t^{\prime}\right)}+\ldots, \\
\mathfrak{g}_{3}\left(t-t^{\prime}\right) & =-\Theta\left(t^{\prime}-t\right) e^{-i \omega_{0}\left(t-t^{\prime}\right)} \\
& -\left(\frac{J}{2}\right)^{2} \int d t^{\prime \prime} \int d \tau \Theta\left(t^{\prime \prime}-t\right) e^{-i \omega_{0}\left(t-t^{\prime \prime}\right)} \\
& \times \bar{G}_{3}\left(t^{\prime \prime}-\tau\right) \Theta\left(t^{\prime}-\tau\right) e^{-i \omega_{0}\left(\tau-t^{\prime}\right)}+\ldots, \\
\mathfrak{g}_{1}^{\prime}\left(t-t^{\prime}\right) & =\Theta\left(t-t^{\prime}\right) e^{-i \omega_{0}\left(t-t^{\prime}\right)} \\
& -\left(\frac{J}{2}\right)^{2} \int d t^{\prime \prime} \int d \tau \Theta\left(t-t^{\prime \prime}\right) e^{-i \omega_{0}\left(t-t^{\prime \prime}\right)} \\
& \times \bar{G}_{1}\left(t^{\prime \prime}-\tau\right) \Theta\left(\tau-t^{\prime}\right) e^{-i \omega_{0}\left(\tau-t^{\prime}\right)}+\ldots,
\end{aligned}
$$

and

$$
\begin{aligned}
\mathfrak{g}_{3}^{\prime}\left(t-t^{\prime}\right) & =\Theta\left(t-t^{\prime}\right) e^{i \omega_{0}\left(t-t^{\prime}\right)} \\
& +\left(\frac{J}{2}\right)^{2} \int d t^{\prime \prime} \int d \tau \Theta\left(t-t^{\prime \prime}\right) e^{i \omega_{0}\left(t-t^{\prime \prime}\right)} \\
& \times \bar{G}_{3}\left(t^{\prime \prime}-\tau\right) \Theta\left(\tau-t^{\prime}\right) e^{i \omega_{0}\left(\tau-t^{\prime}\right)}+\ldots
\end{aligned}
$$

The various Green functions needed for the calculation of the noise spectrum are given here in Fourier space $(\hbar=1)$. The free propagators associated with the fields $Z(t)$ and $z(t)$ are

$$
\begin{aligned}
\mathfrak{g}_{1}^{(0)}(\omega) & =\frac{i}{\omega-\omega_{0}+i \eta} \\
\mathfrak{g}_{1}^{\prime(0)}(\omega) & =\frac{i}{\omega-\omega_{0}-i \eta}
\end{aligned}
$$

In the presence of the conduction electrons they become $(\lambda=J / 2)$, 


$$
\mathfrak{g}_{1}(\omega)=\frac{\mathfrak{g}_{1}^{(0)}(\omega)}{1-\lambda^{2} \bar{G}_{1}(\omega) \mathfrak{g}_{1}^{(0)}(\omega)}
$$

and

$$
\mathfrak{g}_{1}^{\prime}(\omega)=\frac{\mathfrak{g}_{1}^{\prime(0)}(\omega)}{1+\lambda^{2} \bar{G}_{1}(\omega) \mathfrak{g}_{1}^{\prime(0)}(\omega)} .
$$

We will also need their conjugate form, which is again different from the complex conjugate. The free propagator is

$$
\mathfrak{g}_{1}^{\prime *(0)}(\omega)=\frac{i}{\omega+\omega_{0}+i \eta},
$$

and the dressed one is

$$
\mathfrak{g}_{1}^{\prime *}(\omega)=\frac{\mathfrak{g}_{1}^{\prime *(0)}(\omega)}{1-\lambda^{2} \bar{G}_{3}(\omega) \mathfrak{g}_{1}^{\prime *(0)}(\omega)} .
$$

The kernels $\bar{G}_{\alpha},(\alpha=1,4,5)$, are averaged over all spin wave modes. For $\bar{G}_{1}$, it is defined by

$$
\begin{aligned}
\bar{G}_{1}(\omega) & =D \int \frac{d^{3} \mathbf{k}}{(2 \pi)} \int_{-\infty}^{+\infty} \frac{d p}{2 \pi}\left|\frac{\sin \left(p \frac{D}{2}\right)}{p \frac{D}{2}}\right|^{2} \\
& \times\left\{c_{1}^{2} \frac{\partial f}{\partial \varepsilon_{k}}\left(\frac{k_{x} p}{m}+\delta\right) \frac{i}{\delta+\frac{k_{x} p}{m}-\omega+i \eta}\right. \\
& \left.+c_{2}^{2} \frac{\partial f}{\partial \varepsilon_{k}}\left(\frac{-k_{x} p}{m}+\delta\right) \frac{i}{\delta-\frac{k_{x} p}{m}+\omega-i \eta}\right\},
\end{aligned}
$$

with similar definitions for the other kernels. The Fourier transforms are approximately given by

$$
\begin{aligned}
\operatorname{Im} \bar{G}_{1}(\omega) & =v m D \int_{0}^{\infty} \frac{d p}{2 \pi}\left|\frac{\sin \left(p \frac{D}{2}\right)}{p \frac{D}{2}}\right|^{2} \\
& \times\left\{c_{1}^{2}\left(2 \bar{k}_{F}+\frac{m \omega}{p} \log \left[\left|\frac{\delta-\omega+\frac{k_{F} p}{m}}{\delta-\omega-\frac{k_{F} p}{m}}\right|\right]\right)\right. \\
& \left.+c_{2}^{2}\left(2 \bar{k}_{F}+\frac{m \omega}{p} \log \left[\left|\frac{\delta+\omega-\frac{k_{F} p}{m}}{\delta+\omega+\frac{k_{F} p}{m}}\right|\right]\right)\right\},
\end{aligned}
$$

and

$$
\begin{aligned}
\bar{G}_{4}(\omega) & =\frac{m^{2} v}{2} \omega \operatorname{coth}\left(\beta \frac{\omega}{2}\right) D \int_{0}^{\infty} \frac{d p}{\pi p}\left|\frac{\sin \left(p \frac{D}{2}\right)}{p \frac{D}{2}}\right|^{2} \\
& \times\left\{c_{1}^{2} \Theta\left(\bar{k}_{F}-\left|\frac{m(\omega-\delta)}{p}\right|\right)+c_{2}^{2} \Theta\left(\bar{k}_{F}-\left|\frac{m(\omega+\delta)}{p}\right|\right)\right\},
\end{aligned}
$$


and

$$
\begin{aligned}
\bar{G}_{5}(\omega) & =\frac{m^{2} v c_{1} c_{2}}{2} \omega \operatorname{coth}\left(\beta \frac{\omega}{2}\right) D^{2} \int_{0}^{\infty} \frac{d p}{\pi p}\left|\frac{\sin \left(p \frac{D}{2}\right)}{p \frac{D}{2}}\right|^{2} \\
& \times\left\{\Theta\left(\bar{k}_{F}-\left|\frac{m(\omega+\delta)}{p}\right|\right)+\Theta\left(\bar{k}_{F}-\left|\frac{m(\omega-\delta)}{p}\right|\right)\right\} .
\end{aligned}
$$

In all of the above expressions, $\bar{k}_{F}=\frac{k_{F}^{\uparrow}+k_{F}^{\downarrow}}{2}$. It should be also noted that

$$
\bar{G}_{1}^{*}(\omega)=\bar{G}_{1}(-\omega) .
$$

Finally we give the term that is directly responsible for the relaxation term,

$$
\mathcal{R} e \bar{G}_{1}=\frac{\pi m D v}{4} \omega \int \frac{d x}{x}\left(\frac{\sin x}{x}\right)^{2}\left[c_{1}^{2} \Theta\left(\frac{2 k_{F}}{m D} x-|\omega-\Delta|\right)+c_{2}^{2} \Theta\left(\frac{2 k_{F}}{m D} x-|\omega+\Delta|\right)\right],
$$

where $c_{1}^{2}=\left(\sqrt{c_{x x}}+\sqrt{c_{y y}}\right)^{2} /\left(4 \omega_{0}\right)$ and $c_{2}^{2}=\left(\sqrt{c_{x x}}+\sqrt{c_{y y}}\right)^{2} /\left(4 \omega_{0}\right)$. For the noise in the average magnetization, the range of frequencies we are interested in are usually very low compared to the exchange splitting energy. In this case the relaxation time which is proportional to $\mathcal{R} e \bar{G}_{1}$ will depend only on the overall ellipticity factor $c_{1}^{2}+c_{2}^{2}$. The cases of higher frequencies are of interest only in the atomistic limit which will also give a similar expression as in A25 for the relaxation time. In this case, the relaxation as easily seen will depend separately on $c_{x x}$ and $c_{y y}$ and is no longer linear in $\omega$. Memory effects in the systems become important and the GB equation is no longer valid. In the linear model of ref. 4, we did not have any higher order dependence on frequency and the damping was of the Gilbert form. Hence representing a bath by harmonic oscillators is only useful when we are interested in the low energy limit.

* Electronic address: arebei@mailaps.org

1 Gilbert, Phys. Rev. 100, 1243 (1954).

2 W. F. Brown, Jr., Phys. Rev. 130, 1677 (1963).

3 N. Smith, J. Appl. Phys. 90, 5768 (2001).

4 A. Rebei, M. Simionato and G. J. Parker, Phys. Rev. B 69, 134412 (2004).

5 L. Berger, J. App. Phys. 81, 4880 (1997);J. App. Phys. 91, 6795 (2002).

6 Y. Tserkovnyak, A. Brataas, and G. E. W. Bauer, Phys. Rev. Lett. 88, 117601 (2002) 
7 M. D. Stiles and A. Zangwill, Phys. Rev. B, 66, 014407 (2002).

8 E. Simanek and B. Heinrich, Phys. Rev B 67, 144418 (2003).

9 D. L. Mills, Phys. Rev. B 68, 014419 (2003).

10 E. Simanek, Phys. Rev. B 68, 224403 (2003).

11 E. Simanek, cond-mat/0405020 (unpublished).

12 J. C. Slonczewski, J. Magn. Magn. Mater. 159, L1 (1996).

13 D. A. Garanin, Phys. Rev. B 55, 3050 (1997).

14 E. D. Boerner, O. Mryasov, R. W. Chantrell and O. G. Heinonen, 49th Annual Conference on Magnetism and Magnetic Materials, Jacksonville, FL 2004.

15 B. Heinrich, D. Fraitova, and V. Kambersky, Phys. Stat. Sol. 23, 501 (1967).

16 V. Korenman and R. E. Prange, Phys. Rev. B 6, 2769 (1972).

17 J. Kunes and V. Kambersky, Phys. Rev. B 65, 212411 (2002).

18 V. L. Safonov and H. N. Bertram, Phys. Rev. B 65, 172417 (2002).

19 M. Covington et al., Phys. Rev. B 69, 184406 (2004).

20 A. Rebei, (unpublished).

21 C. Kittel, Phys. Rev. 110, 836 (1958).

22 C. Kittel, Quantum Theory of Solids, Wiley, New York 1963.

23 U. Weiss, Quantum dissipative systems, World Scientific, Singapore, 1999.

24 J. Schwinger, J. Math. Phys.2, 407 (1961).

25 J. W. Negele and H. Orland, Quantum Many-Particle Systems, Addison-Wesley, Redwood City, 1988.

26 L. V. Keldysh, Sov. Phys. JETP 20, 1018 (1965).

27 S. Mizukami, Y. Andao, and T. Miyazaki, Phys. Rev. B 66, 104413 (2002).

28 S. M. Bhagat and P. Lubitz, Phys. Rev. B 10, 179 (1974); B. Heinrich, D. J. Meredith, and J. F. Cochran, J. Appl. Phys. 50, 7726 (1979); S. Ingvarsson et al., Phys. Rev. B 66, 214416 (2002).

29 A. O. Caldeira and A. J. Leggett, Physica A 121, 587 (1983).

30 P. C. van Son, H. van Kampen, and P. Wyder, Phys. Rev. Lett. 58, 2271 (1987).

31 M. Zwierzycki, Y. Tserkovnyak, P. J. Kelly, A. Brataas and G. E. Bauer, cond-mat/0402088.

32 A. Brataas, Y. V. Nazarov and G. E. Bauer, Eur. Phys.J. B 22, 99 (2001).

33 A. B. Migdal, JETP 34, 996 (1958). 
34 A. Rebei, W. N. G. Hitchon and R. W. Chantrell, cond-mat/0407051

35 Z. Li and S. Zhang, Phys. Rev. B 69, 134416 (2004).

36 Ya. B. Bazaliy, B. A. Jones, and Shou-Cheng Zhang, Phys. Rev. B 57, R3213 (1998).

37 J. Foros, A. Brataas, G.E.W. Bauer and Y. Tserkovnyak, unpublished.

38 V. Safonov and H. N. Bertram, J. Appl. Phys. 94, 529 (2003).

39 V. Kambersky and C. E. Patton, Phys. Rev. B 11, 2668 (1975).

40 E. M. Ryan et al., 49th Annual Conference on Magnetism and Magnetic Materials, Jacksonville, FL 2004.

41 G. Parker, (unpublished).

42 A. Rebei, L. Berger, R. Chantrell and M. Covington, to appear in J. Appl. Phys.

43 Y. Tserkovnyak, A. Brataas and G. E. Bauer, Phys. Rev.B 67, 140404 (2003). 